This article is published with open access at Springerlink.com

\title{
Galois theory for semiclones
}

\section{Mike Behrisch}

\begin{abstract}
We present a Galois theory connecting finitary operations with pairs of finitary relations, one of which is contained in the other. The Galois closed sets on both sides are characterised as locally closed subuniverses of the full iterative function algebra (semiclones) and relation pair clones, respectively. Moreover, we describe the modified closure operators if only functions and relation pairs of a certain bounded arity, respectively, are considered.
\end{abstract}

\section{Introduction}

Clones of operations, i.e., composition closed sets of operations containing all projections (cf. $[31,35,25,16])$, play an important role in universal algebra, as they encode structural properties independently of the similarity type of the algebra. It is well known (see [10, 15], translations available in [8, 9]) that on finite carrier sets, clones are in a one-to-one correspondence with structures called relational clones. This is established via the Galois correspondence Pol-Inv, which is induced by the relation of "functions preserving relations". In general, i.e., including in particular the case of infinite sets, so-called local closure operators come into play (see [15, 30, 29, 24, 3]), and also the notion of relational clone, as known from finite domains, needs to be generalised (cf. ibid.). In this way, the Galois connection singles out certain locally closed clones from the lattice of all clones on a given set. These clones can also be seen as those which are topologically closed with respect to the topology that one gets by endowing each set $A^{A^{n}}$, for $n \in \mathbb{N}$, with the product topology arising from $A$ initially carrying the discrete topology (see e.g., $[6,5]$ ).

By equipping the set of all finitary functions on a fixed set $A$ with a finite number of operations (including permutation of variables, identification of variables, introduction of fictitious variables, a certain binary composition operation and a projection as a constant; we present more details later on), one obtains the full function algebra of finitary functions on $A$. It is known (cf. e.g., $[31,25])$ that the clones on $A$ are exactly the carrier sets of subalgebras of this structure. This relationship is a special case of the one between the full iterative function algebra, also known as iterative Post algebra (introduced by Mal'cev in [27]), and its subuniverses (called Post algebras in [10]), which

Presented by A. Szendrei.

Received January 20, 2015; accepted in final form January 23, 2016.

2010 Mathematics Subject Classification: Primary: 08A40; Secondary: 08A02, 08A99.

Key words and phrases: iterative algebra, semiclone, relation pair clone, Galois theory. Supported by the Austrian Science Fund (FWF) under grant I836-N23. 
have often only been referred to as closed classes of functions in the Russian literature (e.g., [21, 22]). These are similar in spirit to clones, but they do not need to contain the projections (selectors in the terminology of [27]), as the iterative Post algebra omits the projection constant in its signature compared to the full function algebra.

In analogy to the Pol - Inv Galois connection, there is a Galois correspondence Polp-Invp developed in [17] (see also [18, 19, 20]) based on the notion of functions preserving pairs $\left(\varrho, \varrho^{\prime}\right)$ of relations $\varrho^{\prime} \subseteq \varrho$. For finite carrier sets, the Galois closed sets have been characterised to be precisely the subuniverses of the full iterative Post algebra and the subuniverses of a suitably defined relation pair algebra, respectively. To the best knowledge of the author, a generalisation of this result to arbitrary base sets has not yet appeared in the literature. In particular, the general (and thus infinite) case is also missing in Table 1 of [14, p. 296] summarising related Galois connections and characterisations of their closure operators.

In this article, it is our aim to fill in this gap. We first coin the notion of a semiclone, which relates to transformation semigroups in the same way as clones relate to transformation monoids. It is not hard to figure out that semiclones and subuniverses of the full iterative Post algebra coincide. However, the way a semiclone is defined is much more similar to the usual definition of a clone and easier to grasp than that of a subalgebra of the full iterative function algebra; hence, the proposition of the new terminology of semiclones. Besides, note that our semiclones are different from those appearing in [33].

In a similar fashion as one needed to generalise the notion of relational clone to accommodate the closed sets of $\operatorname{Inv}_{A} \mathrm{Pol}_{A}$ for infinite carrier sets $A$, it will be necessary to modify the relation pair algebra proposed by Harnau in [17]. We shall refer to the corresponding (new) subuniverses as relation pair clones.

Using the same local closure operator as introduced in [15, 29, 30] for sets of functions (the topological closure), and appropriately modifying the local closure on the side of relation pairs, we shall prove the following two main results: the Galois closed sets of operations with respect to $\operatorname{Polp}_{A} \operatorname{Invp}_{A}$ are exactly the locally closed semiclones. Dually, the closed sets of $\operatorname{Invp}_{A} \operatorname{Polp}_{A}$ are precisely the locally closed relation pair clones.

Because it fits nicely in this context, we shall more specifically characterise what it means that a semiclone can be described in the form $\operatorname{Polp}_{A} Q$ for some set $Q$ of less than $s$-ary relation pairs, and that a relation pair clone is given by $\operatorname{Invp}_{A} F$ using a set $F$ of less than $s$-ary operations. As in [30], this involves certain $s$-local closure operators, and so quite a few of our results have analogies in [30], where similar questions have been studied with respect to Pol - Inv.

We mention that a related, in some sense more general, Galois connection has been studied in [28] (finite case) and [13, 12]. There, for fixed sets $A$ and $B$, functions $f: A^{n} \rightarrow B$ have been related to pairs of relations $R \subseteq A^{m}$, $S \subseteq B^{m}$ for some $m \in \mathbb{N}_{+}$, called relational constraints. In this situation, the 
Galois closed sets on the functional side are also closed with respect to variable substitutions (as are our semiclones), but already for syntactic reasons, cannot be closed with respect to compositions. So even if one considers the special case that $B=A$, the results from [13] and [12] describe similar but differently closed sets of functions due to other objects on the dual side (there is no containment condition for the relations as in our setting since for general $A$ and $B$, there cannot be one).

We acknowledge that, perhaps, it could be possible to derive our results by restricting the relational side of the Galois correspondence studied in [13, 12], but we think that the description of the closed objects on the dual side used there is (and has to be) more complicated (using so-called conjunctive minors). Besides, our strategy of proof exhibits more similarities with the classical arguments known from clones and relational clones. Also, the local closures developed for relational constraints in $[13,12]$ seem to require nontrivial modifications (see Remark 2.14) to be used with our relation pairs due to the inclusion requirement in their definition.

A still different weakening of the notion of clone and an associated Galois theory for arbitrary domains has been considered in [26]: there closed sets of functions have been characterised in terms of closed sets of so-called clusters. Since the classes of functions occurring in [26] contain all projections, these results explore a separate direction and cannot be exploited, either, to obtain the missing general (infinite) case for semiclones.

Finally, this article is largely based on a more elaborate version [4] containing explicit proofs of statements that were considered straightforward here.

\section{Preliminaries}

2.1. Notation, functions and relations. In this article, the symbol $\mathbb{N}$ will denote the set of all natural numbers (including zero), and $\mathbb{N}_{+}$will be used for $\mathbb{N} \backslash\{0\}$. Moreover, we shall make use of the standard set theoretic representation of natural numbers by John von Neumann, i.e., $n=\{i \in \mathbb{N} \mid i<n\}$ for $n \in \mathbb{N}$. The power set of a set $S$ will be denoted by $\mathfrak{P}(S)$.

When discussing semiclones, relation pair clones, and their Galois theory, we shall make no further assumptions on the carrier set, which we usually represent by $A$. Any finite (including 0) or infinite cardinality is allowed for $A$.

For sets $A$ and $B$, we write $A^{B}$ for the set of all mappings from $B$ to $A$. The order of composition employed in this article is from right to left, i.e., $g \circ f \in C^{A}$ for $f \in B^{A}$ and $g \in C^{B}$. That is, $g \circ f$ maps elements $a \in A$ to $g(f(a))$. For any index set $I$, sets $A$ and $\left(B_{i}\right)_{i \in I}$, and maps $\left(f_{i}: A \rightarrow B_{i}\right)_{i \in I}$, their tupling is the unique map $h: A \rightarrow \prod_{i \in I} B_{i}$ satisfying $\pi_{i} \circ h=f_{i}$ for each $i \in I$, where $\pi_{i}: \prod_{j \in I} B_{j} \rightarrow B_{i}$ is the $i$-th projection map belonging to the Cartesian product $\prod_{j \in I} B_{j}$. As any ambiguity can usually be resolved from the context, we denote the tupling $h$ by $\left(f_{i}\right)_{i \in I}$, identically to the tuple $\left(f_{i}\right)_{i \in I}$. 
The notion of tupling is, of course, meaningful (by definition) in any category having suitable products, and hence the following simple lemma about composition of tuplings can be proved in such a general context. We recall here just its instance for the category of sets (cf. [3, Lemma 2.5]).

Lemma 2.1. Let $I$ and $J$ be arbitrary index sets, $k, m, n \in \mathbb{N}$ natural numbers, $A, B, D, X$ and $B_{i}($ for $i \in I), C_{j}($ for $j \in J)$ sets. Furthermore, suppose that we are given mappings $r: A \rightarrow B, r_{i}: A \rightarrow B_{i}($ for $i \in I), g_{j}: B \rightarrow C_{j}$ (for $j \in J)$, and $f: \prod_{j \in J} C_{j} \rightarrow D$.

(a) We have $\left(g_{j}\right)_{j \in J} \circ r=\left(g_{j} \circ r\right)_{j \in J}$.

(b) If $B=\prod_{i \in I} B_{i}$, then $\left(g_{j}\right)_{j \in J} \circ\left(r_{i}\right)_{i \in I}=\left(g_{j} \circ\left(r_{i}\right)_{i \in I}\right)_{j \in J}$, and thus

$$
\left(f \circ\left(g_{j}\right)_{j \in J}\right) \circ\left(r_{i}\right)_{i \in I}=f \circ\left(g_{j} \circ\left(r_{i}\right)_{i \in I}\right)_{j \in J} .
$$

(c) If $B_{i}=C_{j}=D=X$ for $i \in I$ and $j \in J, A=X^{k}, I=m$, and $J=n$, then we have

$$
\begin{aligned}
\left(f \circ\left(g_{0}, \ldots, g_{n-1}\right)\right) & \circ\left(r_{0}, \ldots, r_{m-1}\right) \\
& =f \circ\left(g_{0} \circ\left(r_{0}, \ldots, r_{m-1}\right), \ldots, g_{n-1} \circ\left(r_{0}, \ldots, r_{m-1}\right)\right),
\end{aligned}
$$

the superassociativity law for finitary operations on $X$.

In our modelling, natural numbers are simply sets; we consequently interpret tuples as maps, too: if $B=n \in \mathbb{N}$ is a natural number, then $A^{B}=A^{n}$ is the set of all $n$-tuples $x=(x(i))_{i<n}$. We shall often write $x_{i}$ for the entry $x(i)$ (for $i \in n$ ), and whenever convenient, we shall also refer to the entries of tuples by different indexing, e.g., $x=\left(x_{1}, \ldots, x_{n}\right)$. Note that the sole element of $A^{0}=A^{\emptyset}$ is the empty mapping (tuple), whose graph is the empty relation. It will consistently be denoted by $\emptyset$. As tuples are functions, we may compose them with other functions, e.g., if $x \in A^{n}$ and $\alpha: m \rightarrow n$, (for $m, n \in \mathbb{N}$ ), then $x \circ \alpha$ is the tuple in $A^{m}$ whose entries are $x_{\alpha(i)}$ (for $i \in m$ ). Similarly, if $g: A \rightarrow B$, then $g \circ x=\left(g\left(x_{i}\right)\right)_{i \in n}$ is an element of $B^{n}$.

Any mapping $f \in A^{A^{n}}$ (for $n \in \mathbb{N}$ ) is called an $n$-ary operation on $A$, and the number $n$ is referred to as its arity, denoted by $\operatorname{ar}(f)$. The set of all finitary operations on $A$ is $\mathrm{O}_{A}:=\biguplus_{k \in \mathbb{N}} A^{A^{k}}$. Note that we explicitly include nullary operations here, which is slightly uncommon in standard clone theory. For a set of operations $F \subseteq \mathrm{O}_{A}$, we denote its $n$-ary part by $F^{(n)}:=F \cap A^{A^{n}}$. We extend this notation to operators yielding subsets of operations: for an operator on a set $S, \mathrm{OP}: S \rightarrow \mathfrak{P}\left(\mathrm{O}_{A}\right)$, we define $\mathrm{OP}^{(n)}: S \rightarrow \mathfrak{P}\left(\mathrm{O}_{A}^{(n)}\right)$ by the restriction $\operatorname{OP}^{(n)}(s):=(\operatorname{OP}(s))^{(n)}$ for $s \in S$. Based on this, we put moreover $\mathrm{OP}^{\left(n_{1}, \ldots, n_{k}\right)}(s):=\biguplus_{i=1}^{k} \mathrm{OP}^{\left(n_{i}\right)}(s)$ for $s \in S$ and a finite list of arities $n_{1}, \ldots, n_{k}, k>0$. We also abbreviate $\mathrm{OP}^{(0, \ldots, n)}$ as $\mathrm{OP}^{(\leq n)}$ or $\mathrm{OP}^{(<n+1)}$, and for $s \in S$, we let $\mathrm{OP}^{(>0)}(s):=\mathrm{OP}(s) \backslash \mathrm{OP}^{(0)}(s)$; moreover, $\mathrm{OP}^{\left(<\aleph_{0}\right)}:=\mathrm{OP}$.

The projection operations belonging to the finite Cartesian powers of the carrier set play a special role. For $n \in \mathbb{N}$ and $i \in n$, we denote by $e_{i}^{(n)} \in \mathrm{O}_{A}^{(n)}$ 
the $n$-ary projection on the $i$-th coordinate. Evidently, there do not exist any nullary projections. Therefore, the set of all projections on $A$, denoted by $\mathrm{J}_{A}$, equals $\bigcup_{n \in \mathbb{N}_{+}}\left\{e_{i}^{(n)} \mid 0 \leq i<n\right\}$. For the identity operation $e_{0}^{(1)}$, we occasionally also use the notation $\mathrm{id}_{A}$.

Knowing about re-indexing tuples, we can recollect the notion of polymer. If $m, n \in \mathbb{N}$ are arities, $\alpha: n \rightarrow m$ is any indexing map, and $f \in \mathrm{O}_{A}^{(n)}$, then $\delta_{\alpha}(f)$ is the operation in $\mathrm{O}_{A}^{(m)}$ given by $\delta_{\alpha}(f)(x):=f(x \circ \alpha)$ for $x \in A^{m}$. Any operation derived from $f \in \mathrm{O}_{A}^{(n)}$ by some map $\alpha: n \rightarrow m$, (for $m \in \mathbb{N}$ ), is said to be a polymer of $f$. Clearly, any polymer of $f$ can be obtained by composition with a suitable tupling of projections: $\delta_{\alpha}(f)=f \circ\left(e_{\alpha(i)}^{(m)}\right)_{i \in n}$.

Besides operations, we shall also need relations: for $m \in \mathbb{N}$, any subset $\varrho \subseteq A^{m}$ of $m$-tuples is an $m$-ary relation on $A$. Thus, $\mathfrak{P}\left(A^{m}\right)$ is the set of all $m$-ary relations, and again allowing arity equal to null, the set of all finitary relations is defined by $\mathrm{R}_{A}:=\bigcup_{\ell \in \mathbb{N}} \mathfrak{P}\left(A^{\ell}\right)$. If $Q \subseteq \mathrm{R}_{A}$, we use $Q^{(m)}:=Q \cap \mathfrak{P}\left(A^{m}\right)$ to denote its $m$-ary part. Moreover, if $\mathrm{OP}: S \rightarrow \mathfrak{P}\left(\mathrm{R}_{A}\right)$ is an operator on a set $S$, we put $\mathrm{OP}^{(m)}: S \rightarrow \mathfrak{P}\left(\mathrm{R}_{A}^{(m)}\right)$, mapping $s \in S$ to $\mathrm{OP}^{(m)}(s):=(\mathrm{OP}(s))^{(m)}$. Similarly as for operations, for $s \in S$, we define $\mathrm{OP}^{(<m+1)}(s):=\bigcup_{k=0}^{m} \mathrm{OP}^{(k)}(s), \mathrm{OP}^{(\geq m)}(s):=\bigcup_{k \in \mathbb{N}, k \geq m} \mathrm{OP}^{(k)}(s)$, and we let $\mathrm{OP}^{(>0)}:=\mathrm{OP}^{(\geq 1)}$, as well as $\mathrm{OP}^{\left(<\aleph_{0}\right)}:=\mathrm{OP}$.

A relation pair of arity $m \in \mathbb{N}\left([17\right.$, p. 15$]$ or $\left[19\right.$, p. 11]) is any pair $\left(\varrho, \varrho^{\prime}\right)$, where $\varrho, \varrho^{\prime} \in \mathrm{R}_{A}^{(m)}$ and $\varrho^{\prime} \subseteq \varrho$. We collect all $m$-ary relation pairs in the set $\mathrm{Rp}_{A}^{(m)}$; the disjoint union (the importance of this technical aspect is discussed after Definition 2.8) $\mathrm{Rp}_{A}:=\biguplus_{\ell \in \mathbb{N}} \mathrm{Rp}_{A}^{(\ell)}$ denotes the set of all finitary relation pairs. As before, we abbreviate $m$-ary parts as $Q^{(m)}:=\operatorname{Rp}_{A}^{(m)} \cap Q$ for any $Q \subseteq \mathrm{Rp}_{A}$ and define operator restrictions $\mathrm{OP}^{(m)}: S \rightarrow \mathfrak{P}\left(\operatorname{Rp}_{A}^{(m)}\right)$ by mapping $s \in S$ to $\mathrm{OP}^{(m)}(s):=(\mathrm{OP}(s))^{(m)}$ for any $\mathrm{OP}: S \rightarrow \mathfrak{P}\left(\mathrm{Rp}_{A}\right)$. Further, we put $\mathrm{OP}^{(<m+1)}(s):=\biguplus_{k=0}^{m} \mathrm{OP}^{(k)}(s)$ for $s \in S$ and $\mathrm{OP}^{\left(<\aleph_{0}\right)}:=\mathrm{OP}$.

There is a natural order relation on $\operatorname{Rp}_{A}^{(m)}$ for each $m \in \mathbb{N}$, which is given by set inclusion in both components. That is, we write $\left(\sigma, \sigma^{\prime}\right) \leq\left(\varrho, \varrho^{\prime}\right)$ for $\left(\varrho, \varrho^{\prime}\right),\left(\sigma, \sigma^{\prime}\right) \in \operatorname{Rp}_{A}^{(m)}$ if and only if $\sigma \subseteq \varrho$ and $\sigma^{\prime} \subseteq \varrho^{\prime}$. Moreover, we shall need the quasiorder on $\operatorname{Rp}_{A}^{(m)}$, (for $m \in \mathbb{N}$ ), that is specified by just ordering the first components: $\left(\sigma, \sigma^{\prime}\right) \preceq\left(\varrho, \varrho^{\prime}\right)$ holds by definition if and only if $\sigma \subseteq \varrho$.

We say that a relation pair $\left(\sigma, \sigma^{\prime}\right) \in \mathrm{Rp}_{A}$ is a relaxation of some other pair $\left(\varrho, \varrho^{\prime}\right) \in \operatorname{Rp}_{A}$ (cf. [13, p. 153]) if $\varrho^{\prime} \subseteq \sigma^{\prime}$ and $\sigma \subseteq \varrho$. A collection $Q \subseteq \operatorname{Rp}_{A}$ is closed with respect to relaxations if with each pair $\left(\varrho, \varrho^{\prime}\right) \in Q$, it also contains any of its relaxations, i.e., if

$$
\rightarrow Q^{\leftarrow}:=\left\{\left(\sigma, \sigma^{\prime}\right) \in \operatorname{Rp}_{A} \mid \exists\left(\varrho, \varrho^{\prime}\right) \in Q: \varrho^{\prime} \subseteq \sigma^{\prime} \subseteq \sigma \subseteq \varrho\right\}
$$

is a subset of (equal to) $Q$. Since set inclusion is transitive, the collection $\rightarrow Q^{\leftarrow}$ is the least subset of $\operatorname{Rp}_{A}$ (with respect to $\subseteq$ ) that contains $Q$ and is closed with respect to relaxations. We call $\rightarrow Q^{\leftarrow}$ the closure of $Q$ with respect 
to relaxation. In [19, Definition 1, p. 16], this closure has been handled by socalled multioperations $d_{\mathrm{v}}$ and $d_{\mathrm{h}}$.

2.2. The Galois correspondence Polp-Invp. Here we recall the Galois connection Polp-Invp as defined in [17, p. 15] and [19, p. 11]. We slightly extend the scope by allowing nullary operations and relations.

Definition 2.2. For an $n$-ary operation $f \in \mathrm{O}_{A}^{(n)}(n \in \mathbb{N})$ and an $m$-ary relation pair $\left(\varrho, \varrho^{\prime}\right) \in \operatorname{Rp}_{A}^{(m)}$ (for $m \in \mathbb{N}$ ) on a set $A$, we say that $f$ preserves $\left(\varrho, \varrho^{\prime}\right)$ and write $f \triangleright\left(\varrho, \varrho^{\prime}\right)$ if the following equivalent conditions hold.

(i) For every tuple $\mathbf{r} \in \varrho^{n}$, the composition of $f$ with the tupling (r) of the tuples in $\mathbf{r}$ belongs to the smaller relation: $f \circ(\mathbf{r}) \in \varrho^{\prime}$.

(ii) For every $(m \times n)$-matrix $X \in A^{m \times n}$ whose columns $X_{-, j}$ (for $j \in n$ ) are tuples in $\varrho$, the tuple $\left(f\left(X_{i,-}\right)\right)_{i \in m}$ obtained by row-wise application of $f$ to $X$ yields a tuple of $\varrho^{\prime}$.

Note, in this respect, that for any tuple $\mathbf{r}=\left(r_{j}\right)_{0<j<n} \in\left(A^{m}\right)^{n}$, where for $0 \leq j<n$, each tuple is given as $r_{j}=\left(r_{i j}\right)_{0<i<m}$, the definition of tupling precisely yields that $f \circ(\mathbf{r})=\left(f\left(\left(r_{i j}\right)_{0 \leq j<n}\right)\right)_{0 \leq i<m}$, i.e., the result of applying $f$ row-wise to the matrix $\left(r_{i j}\right)_{(i, j) \in m \times n} \in A^{m \times n}$.

Note, furthermore, that for $\varrho \in \mathrm{R}_{A}$ and $f \in \mathrm{O}_{A}$, the condition $f \triangleright(\varrho, \varrho)$ coincides with the usual preservation condition for functions and relations (cf. [3, Definition 2.3] for the framework involving nullary operations).

Based on the preservation condition we introduce a Galois correspondence in the usual way: for a set $F \subseteq \mathrm{O}_{A}$, we denote by

$$
\operatorname{Invp}_{A} F:=\left\{\left(\varrho, \varrho^{\prime}\right) \in \operatorname{Rp}_{A} \mid \forall f \in F: f \triangleright\left(\varrho, \varrho^{\prime}\right)\right\}
$$

the set of its invariant relation pairs, and, dually, for $Q \subseteq \mathrm{R}_{A}$, the set

$$
\operatorname{Polp}_{A} Q:=\left\{f \in \mathrm{O}_{A} \mid \forall\left(\varrho, \varrho^{\prime}\right) \in Q: f \triangleright\left(\varrho, \varrho^{\prime}\right)\right\}
$$

contains all polymorphisms of relation pairs in $Q$. The pair $\left(\operatorname{Polp}_{A}, \operatorname{Invp}_{A}\right)$ forms the Galois correspondence Polp-Invp.

If we restrict the latter just to relation pairs $\left(\varrho, \varrho^{\prime}\right)$ where $\varrho=\varrho^{\prime}$, then we get the standard Galois connection Pol-Inv: for $F \subseteq \mathrm{O}_{A}$, we have

$$
\left\{(\varrho, \varrho) \mid \varrho \in \mathrm{R}_{A} \wedge \forall f \in F: f \triangleright(\varrho, \varrho)\right\}=\left\{(\varrho, \varrho) \mid \varrho \in \operatorname{Inv}_{A} F\right\},
$$

where $\operatorname{Inv}_{A} F=\left\{\varrho \in \mathrm{R}_{A} \mid \forall f \in F: f \triangleright \varrho\right\}$; and for $Q \subseteq\left\{(\varrho, \varrho) \mid \varrho \in \mathrm{R}_{A}\right\}$, letting $Q^{\prime}:=\left\{\varrho \in \mathrm{R}_{A} \mid(\varrho, \varrho) \in Q\right\}$, it is the case that

$$
\operatorname{Polp}_{A} Q=\left\{f \in \mathrm{O}_{A} \mid \forall(\varrho, \varrho) \in Q: f \triangleright(\varrho, \varrho)\right\}=\mathrm{Pol}_{A} Q^{\prime},
$$

wherein $\mathrm{Pol}_{A} Q^{\prime}:=\left\{f \in \mathrm{O}_{A} \mid \forall \varrho \in Q^{\prime}: f \triangleright \varrho\right\}$.

The name polymorphism attributed to the functions in $\operatorname{Pol}_{A} Q$ for sets of relations $Q \subseteq \mathrm{R}_{A}$ comes from the fact that an operation $f \in \mathrm{O}_{A}$ belongs to 
$\mathrm{Pol}_{A} Q$ if and only if it is a homomorphism from the power $\underbrace{\mathbf{a r}(f)}$ into the relational structure $\underset{\sim}{\mathbf{A}}=\left\langle A ;(\varrho)_{\varrho \in Q}\right\rangle$. This characterisation can be generalised.

Lemma 2.3. For $Q \subseteq \operatorname{Rp}_{A}$ and any arity $n \in \mathbb{N}$, an operation $f \in \mathrm{O}_{A}^{(n)}$ satisfies $f \in \operatorname{Polp}_{A} Q$ if and only if $f:\left\langle A ;(\varrho)_{\left(\varrho, \varrho^{\prime}\right) \in Q}\right\rangle^{n} \rightarrow\left\langle A ;\left(\varrho^{\prime}\right)_{\left(\varrho, \varrho^{\prime}\right) \in Q}\right\rangle$ is a homomorphism of relational structures.

It is an evident consequence of the definition of preservation that sets of the form $\operatorname{Invp}_{A} F$, for $F \subseteq \mathrm{O}_{A}$, are closed with respect to relaxation (cf. [19, Lemma 8, p. 16]).

Lemma 2.4. For $F \subseteq \mathrm{O}_{A}$, we have $\operatorname{Invp}_{A} F=\operatorname{Invp}_{A} F^{\leftarrow}$.

Corollary 2.5. For every $Q \subseteq \operatorname{Rp}_{A}$, we have $\operatorname{Polp}_{A} Q=\operatorname{Polp}_{A} \rightarrow Q^{\leftarrow}$.

Proof. By Lemma 2.4, $Q \subseteq \rightarrow Q^{\leftarrow} \subseteq \rightarrow \operatorname{Invp}_{A} \operatorname{Polp}_{A} Q^{\leftarrow}=\operatorname{Invp}_{A} \operatorname{Polp}_{A} Q$ for $Q \subseteq \mathrm{Rp}_{A}$, so $\operatorname{Polp}_{A} Q \supseteq \operatorname{Polp}_{A} \rightarrow Q^{\leftarrow} \supseteq \operatorname{Polp}_{A} \operatorname{Invp}_{A} \operatorname{Polp}_{A} Q=\operatorname{Polp}_{A} Q$.

If a set $Q \subseteq \mathrm{Rp}_{A}$ or, more generally, $\operatorname{Invp}_{A} \operatorname{Polp}_{A} Q$ contains a relation pair that is not preserved by any operation, then $\operatorname{Polp}_{A} Q$ is forced to be empty. The next lemma characterises when this happens (cf. [17, p. 15] and [19, p. 12]).

Lemma 2.6. For $Q \subseteq \operatorname{Rp}_{A}$, we have $\operatorname{Polp}_{A} Q=\emptyset$ if and only if $\operatorname{Invp}_{A} \operatorname{Polp}_{A} Q$ contains a relation pair of the form $(\varrho, \emptyset)$ with $\varrho \neq \emptyset$, which happens precisely if $\left(A^{0}, \emptyset\right) \in \operatorname{Invp}_{A} \operatorname{Polp}_{A} Q$.

Remark 2.7. The previous lemma demonstrates the necessity to include nullary relations in the framework, caused by our wish not to impose any restriction on the carrier set $A$. Namely, for $A=\emptyset$, we have $A^{m}=\emptyset$ for all $m \in \mathbb{N}_{+}$, and thus $\mathrm{R}_{A}^{(m)}=\mathfrak{P}\left(A^{m}\right)=\{\emptyset\}$. Hence, $\operatorname{Rp}_{A}=\left\{\left(A^{0}, \emptyset\right)\right\} \uplus \biguplus_{m \in \mathbb{N}_{+}}\{(\emptyset, \emptyset)\}$, which allows us to distinguish between $\operatorname{Polp}_{A} \operatorname{Rp}_{A}=\operatorname{Polp}_{A}\left\{\left(A^{0}, \emptyset\right)\right\}=\emptyset$ and $\operatorname{Polp}_{A}\{(\emptyset, \emptyset)\}=\operatorname{Polp}_{A} \emptyset=\mathrm{O}_{A}$. Both sets are evidently semiclones (subalgebras of the iterative Post algebra) on any carrier set $A$, so in view of our overall objective, it is more than desirable to be able to model them with our Galois correspondence. Restricting to relations of positive arity, this would clearly be impossible for $A=\emptyset$.

2.3. Local closure operators for functions and relation pairs. For the Galois connection Pol-Inv in the case of infinite carrier sets, there exist examples $F \subseteq \mathrm{O}_{A}$ where the inclusion $\langle F\rangle_{\mathrm{O}_{A}} \subseteq \mathrm{Pol}_{A} \operatorname{Inv}_{A} F$ is proper. Hence, in order to characterise the Galois closure, an additional local closure operator is needed. A similar situation arises with Polp-Invp: for operations, we can indeed reuse the same local closure operators as known from Pol-Inv. For the side of relation pairs, we have to introduce a new variant of local closure.

In fact, in order to characterise Galois closures of sets of less than $s$-ary operations/relations (for $s \in \mathbb{N}_{+}$), we define more specific variants of $s$-local 
closure operators. Note the following change of indexing compared to the literature: for $s \geq 2$, our operator $s-\operatorname{Loc}_{A}$ coincides with the operator $(s-1)-\operatorname{Loc}_{A}$ from $\left[30,1.9\right.$, p. 15] (see also [29, 1.5, p. 255 et seq.]); $\aleph_{0}-\operatorname{Loc}_{A}$ is known as $\operatorname{Loc}_{A}$.

Definition 2.8. For a cardinal $0<s \leq \aleph_{0}, F \subseteq \mathrm{O}_{A}$, and $Q \subseteq \mathrm{Rp}_{A}$, we set

$$
\begin{array}{r}
s-\operatorname{Loc}_{A} F:=\biguplus_{n \in \mathbb{N}}\left\{g \in \mathrm{O}_{A}^{(n)}\left|\forall B \subseteq A^{n},\right| B\left|<s \exists f \in F^{(n)}: g\right|_{B}=\left.f\right|_{B}\right\}, \\
s-\operatorname{LOC}_{A} Q:=\biguplus_{m \in \mathbb{N}}\left\{\left(\sigma, \sigma^{\prime}\right) \in \operatorname{Rp}_{A}^{(m)} \mid \begin{array}{r}
\forall \subseteq \sigma,|B|<s \exists\left(\varrho, \varrho^{\prime}\right) \in Q^{(m)}: \\
B \subseteq \varrho \wedge \varrho^{\prime} \subseteq \sigma^{\prime}
\end{array}\right\},
\end{array}
$$

and call these $s$-local closure operators. For compatibility with traditional use, we call $\aleph_{0}-\mathrm{Loc}_{A}$ and $\aleph_{0}-\mathrm{LOC}_{A}$ local closure operators.

It is easy to check that $s-\mathrm{Loc}_{A}$ and $s-\mathrm{LOC}_{A}$ are indeed closure operators on $\mathrm{O}_{A}$ and $\mathrm{Rp}_{A}$, respectively. Moreover, $\aleph_{0}-\operatorname{Loc}_{A} F=\bigcap_{s \in \mathbb{N}_{+}} s-\operatorname{Loc}_{A} F$ and $\aleph_{0}-\mathrm{LOC}_{A} Q=\bigcap_{s \in \mathbb{N}_{+}} s-\mathrm{LOC}_{A} Q$ hold for $F \subseteq \mathrm{O}_{A}$ and $Q \subseteq \mathrm{Rp}_{A}$. Likewise, it is not hard to see that for every $n \in \mathbb{N}$ and cardinals $0<s \leq \aleph_{0}$, we have $s-\operatorname{Loc}_{A}^{(n)} F=s-\operatorname{Loc}_{A}\left(F^{(n)}\right)$ for $F \subseteq \mathrm{O}_{A}$, and $s-\operatorname{LOC}_{A}^{(n)} Q=s-\operatorname{LOC}_{A}\left(Q^{(n)}\right)$ for any set $Q \subseteq \mathrm{Rp}_{A}$. To make a technical remark: if we had not insisted on using the disjoint union for the definition of $\mathrm{Rp}_{A}$, then for any $n \in \mathbb{N}$, we would have $1-\operatorname{LOC}_{A}\left(Q^{(n)}\right)=\operatorname{Rp}_{A}$ whenever $(\emptyset, \emptyset) \in Q$ (as in this case, $(\emptyset, \emptyset) \in Q^{(n)^{(m)}}$ were true for all $\left.m \in \mathbb{N}\right)$, and this would obviously violate the equality mentioned above: $\operatorname{Rp}_{A}=1-\mathrm{LOC}_{A}\left(Q^{(n)}\right) \nsubseteq 1-\mathrm{LOC}_{A}^{(n)} Q \subseteq \operatorname{Rp}_{A}^{(n)}$.

Moreover, it follows directly from the definition that $t-\operatorname{Loc}_{A} F \subseteq s-\operatorname{Loc}_{A} F$ and $t-\mathrm{LOC}_{A} Q \subseteq s-\mathrm{LOC}_{A} Q$ hold for all sets $F \subseteq \mathrm{O}_{A}$ and $Q \subseteq \mathrm{Rp}_{A}$ whenever $0<s \leq t \leq \aleph_{0}$. Thus, for $F \subseteq \mathrm{O}_{A}, Q \subseteq \mathrm{Rp}_{A}$ and $s \in \mathbb{N}_{+}$, we have

$$
\begin{aligned}
& 1-\operatorname{Loc}_{A} F \supseteq \cdots \supseteq s-\operatorname{Loc}_{A} F \supseteq(s+1)-\operatorname{Loc}_{A} F \supseteq \cdots \supseteq \aleph_{0}-\operatorname{Loc}_{A} F \supseteq F \text {, } \\
& 1-\mathrm{LOC}_{A} Q \supseteq \cdots \supseteq s-\mathrm{LOC}_{A} Q \supseteq(s+1)-\mathrm{LOC}_{A} Q \supseteq \cdots \supseteq \aleph_{0}-\mathrm{LOC}_{A} Q \supseteq Q \text {. }
\end{aligned}
$$

It follows from these relations that

$$
s-\operatorname{Loc}_{A} t-\operatorname{Loc}_{A} F=(\min \{s, t\})-\operatorname{Loc}_{A} F
$$

holds for all $F \subseteq \mathrm{O}_{A}$ and

$$
s-\mathrm{LOC}_{A} t-\mathrm{LOC}_{A} Q=(\min \{s, t\})-\mathrm{LOC}_{A} Q
$$

for all $Q \subseteq \operatorname{Rp}_{A}$ and any $0<s, t \leq \aleph_{0}$ (cp. [30, Proposition 1.10, p. 16]).

Note that our definition of $s$-local closure of relation pairs for $s \geq 2$ entails the corresponding one for relations given in $[30,1.9, \mathrm{p} .16]$ in the following way: for $Q^{\prime} \subseteq \mathrm{R}_{A} \backslash \mathrm{R}_{A}^{(0)}$, put $Q:=\biguplus_{m \in \mathbb{N}_{+}}\left\{(\varrho, \varrho) \mid \varrho \in Q^{\prime(m)}\right\}$. Then given $1<s \leq \aleph_{0}$, one can verify $s-\mathrm{LOC}_{A} Q=\biguplus_{m \in \mathbb{N}_{+}}\left\{(\sigma, \sigma) \mid \sigma \in s-\mathrm{L} \varnothing \mathrm{C}_{A}^{(m)} Q^{\prime}\right\}$ (see Lemma 6.13 for further details), in which $s$-L $\varnothing \mathrm{C}_{A} Q^{\prime}$ denotes the set $\left\{\sigma \in \mathrm{R}_{A}|\forall B \subseteq \sigma| B \mid,<s \exists \varrho \in Q^{\prime}: B \subseteq \varrho \subseteq \sigma\right\}$. Hence, one may reconstruct 
$s-\mathrm{L} \varnothing \mathrm{C}_{A} Q^{\prime}$ as $\left\{\sigma \in \mathrm{R}_{A} \mid(\sigma, \sigma) \in s-\mathrm{LOC}_{A} Q\right\}$. For $1<s<\aleph_{0}$, the operator $s$-L $\varnothing \mathrm{C}_{A}$ was called $(s-1)-\mathrm{LOC}_{A}$ in [30], and $\mathrm{LOC}_{A}$ was used for $\aleph_{0}-\mathrm{L} \varnothing \mathrm{C}_{A}$.

The following is an obvious consequence of Definition 2.8.

Lemma 2.9. For $Q \subseteq \mathrm{Rp}_{A}$, we have $\rightarrow Q^{\leftarrow} \subseteq \aleph_{0}-\mathrm{LOC}_{A} Q$.

Corollary 2.10. Let $Q \subseteq \mathrm{Rp}_{A}$ be s-locally closed for some $0<s \leq \aleph_{0}$; then it is closed with respect to relaxation.

For relations of fixed arity and finite base sets, there is even a much stronger connection between relaxation and $s$-local closure.

Lemma 2.11. For all finite carrier sets $A$ of cardinality $k:=|A|<\aleph_{0}$ and any $m \in \mathbb{N}$, we have $\rightarrow Q^{\leftarrow}=\aleph_{0}-\mathrm{LOC}_{A} Q=\left(k^{m}+1\right)-\mathrm{LOC}_{A} Q$ for all $Q \subseteq \mathrm{Rp}_{A}^{(m)}$.

Proof. Let $\ell:=k^{m}+1$. The inclusions $\rightarrow Q^{\leftarrow} \subseteq \aleph_{0}-\mathrm{LOC}_{A} Q \subseteq \ell-\mathrm{LOC}_{A} Q$ hold in general (cf. Lemma 2.9). Conversely, for a set $Q \subseteq \mathrm{Rp}_{A}^{(m)}$ of $m$-ary pairs, let us consider any $\left(\sigma, \sigma^{\prime}\right) \in \ell-\mathrm{LOC}_{A} Q=\ell-\mathrm{LOC}_{A}\left(Q^{(m)}\right)=\ell-\mathrm{LOC}_{A}^{(m)} Q$. As $\sigma \in \mathrm{R}_{A}^{(m)}$, we have $|\sigma| \leq\left|A^{m}\right|<\ell$. Hence, taking $B:=\sigma$ as a subset of $\sigma$ having less than $\ell$ elements, by definition of $\ell-\mathrm{LOC}_{A}$, there is $\left(\varrho, \varrho^{\prime}\right) \in Q^{(m)}$ such that $\sigma=B \subseteq \varrho$ and $\varrho^{\prime} \subseteq \sigma^{\prime}$. Therefore, $\left(\sigma, \sigma^{\prime}\right) \in \rightarrow Q^{\leftarrow}$.

Corollary 2.12. For finite $A$, we have $\rightarrow Q^{\leftarrow}=\aleph_{0}-\mathrm{LOC}_{A} Q$ for all $Q \subseteq \mathrm{Rp}_{A}$; thus, $Q \subseteq \mathrm{Rp}_{A}$ is locally closed if and only if it is closed with respect to relaxation.

The following closure property will become important regarding the characterisation of the closure operator $\operatorname{Invp}_{A} \operatorname{Polp}_{A}^{(<s)}$ in Section 5 . For $0<s \leq \aleph_{0}$, a collection $\mathcal{T} \subseteq \mathfrak{P}(S)$ of subsets of a set $S$ is called $s$-directed if and only if for all $0 \leq t<s$, all $\left(X_{i}\right)_{i \in t} \in \mathcal{T}^{t}$, and every $\mathbf{r}=\left(r_{i}\right)_{i \in t} \in \prod_{i \in t} X_{i}$, there is a set $Z \in \mathcal{T}$ such that $\operatorname{im} \mathbf{r}=\left\{r_{i} \mid i \in t\right\} \subseteq Z$. For $s<\aleph_{0}$, this condition is equivalent to $\mathcal{T}$ being non-empty and that for all $\left(X_{i}\right)_{0<i<s-1} \in \mathcal{T}^{s-1}$ and $\mathbf{r} \in \prod_{0 \leq i<s-1} X_{i}$, there is $Z \in \mathcal{T}$ fulfilling $\operatorname{im} \mathbf{r} \subseteq Z$. Certainly, $\mathcal{T} \subseteq \mathfrak{P}(S)$ is $\aleph_{0}$-directed if and only if it is $s$-directed for all $0<s<\aleph_{0}$. We say that a set $Q \subseteq \operatorname{Rp}_{A}^{(m)}$ of $m$-ary relation pairs is $s$-directed if and only if $\left\{\varrho \mid\left(\varrho, \varrho^{\prime}\right) \in Q\right\} \subseteq \mathfrak{P}\left(A^{m}\right)$ is $s$-directed in the sense above. Sets of the form $s$-LOC $A$, where $Q \subseteq \mathrm{Rp}_{A}$, are closed with respect to unions of $s$-directed systems of relation pairs of the same arity.

Lemma 2.13. If for $0<s \leq \aleph_{0}, m \in \mathbb{N}$, and $Q \subseteq \mathrm{Rp}_{A}$, a set $\mathcal{T} \subseteq s-\mathrm{LOC}_{A}^{(m)} Q$ is s-directed, then we have $\bigcup \mathcal{T}:=\left(\bigcup_{\left(\mu, \mu^{\prime}\right) \in \mathcal{T}} \mu, \bigcup_{\left(\mu, \mu^{\prime}\right) \in \mathcal{T}} \mu^{\prime}\right) \in s-\mathrm{LOC}_{A}^{(m)} Q$.

We call a set $\mathcal{T} \subseteq \operatorname{Rp}_{A}^{(m)}$ directed if $\mathcal{T} \neq \emptyset$ and for all $\left(\varrho_{1}, \varrho_{1}^{\prime}\right),\left(\varrho_{2}, \varrho_{2}^{\prime}\right) \in \mathcal{T}$, there exists some $\left(\varrho, \varrho^{\prime}\right) \in \mathcal{T}$ such that $\varrho_{1} \cup \varrho_{2} \subseteq \varrho$. This is equivalent to saying that for any finite subset $\mathcal{F} \subseteq \mathcal{T}$, there is a pair $\left(\varrho, \varrho^{\prime}\right) \in \mathcal{T}$ such that $\bigcup_{\left(\mu, \mu^{\prime}\right) \in \mathcal{F}} \mu \subseteq \varrho$, wherefore directedness clearly implies $\aleph_{0}$-directedness. 
As a consequence of this implication, we get that locally closed sets of relation pairs are closed under directed unions of sets of pairs of identical arity.

Under additional assumptions on the set of relation pairs $Q$, we shall extend Lemma 2.13 to characterisations of $s$-local closedness for $0<s \leq \aleph_{0}$. We conclude this subsection with remarks on the relationship of our local closure operators to others defined in the more general setting in [12].

Remark 2.14. The $s$-local closure operators for $0<s \leq \aleph_{0}$ defined here cannot directly be derived as special cases of the corresponding closure operators from [12]. As the case of local closures is similar, we shall only argue for $s$-local closures. Specialising the framework in the mentioned article for a pair of carrier sets $(A, B)$ where $B=A$, we may apply the $s$-local closure $\mathbf{L} \mathbf{O}_{s}$ described there (for $1 \leq s<\aleph_{0}$ ) to any set $Q \subseteq \mathrm{Rp}_{A} \backslash \mathrm{Rp}_{A}^{(0)}$, then yielding the collection $\mathbf{L O}_{s}(Q)=Q \cup Q^{\prime}$ where

$$
\begin{aligned}
& Q^{\prime}:=\bigcup_{m \in \mathbb{N}_{+}}\left\{(R, S) \in\left(\mathfrak{P}\left(A^{m}\right)\right)^{2} \mid\right. \\
&\left.\forall C \subseteq R,|C| \leq s \forall A^{m} \supseteq T \supseteq S:(C, T) \in Q\right\} .
\end{aligned}
$$

As this set contains pairs $(R, S)$ that are not relation pairs, i.e., failing the condition $R \supseteq S$, the canonical modification would be to simply intersect the result with $\operatorname{Rp}_{A}$, leading to $\mathbf{L O}_{s}(Q) \cap \mathrm{Rp}_{A}=Q \cup Q^{\prime \prime}$ with

$$
\begin{aligned}
& Q^{\prime \prime}:=\bigcup_{m \in \mathbb{N}_{+}}\left\{(R, S) \in \operatorname{Rp}_{A}^{(m)} \mid\right. \\
&\left.\forall C \subseteq R,|C| \leq s \forall A^{m} \supseteq T \supseteq S:(C, T) \in Q\right\} .
\end{aligned}
$$

This set $Q \cup Q^{\prime \prime}$ equals $Q$ on any set $A$ (in fact, $Q^{\prime \prime}=\emptyset$, whenever $A \neq \emptyset$ ). So $\mathbf{L O}_{s}$ (or its canonical modification) is not helpful at all in our setting.

Suppose, in the union over $m \in \mathbb{N}_{+}$, we change the condition describing when a relation pair $(R, S)$ is added to the $s$-local closure of $Q$ as follows: among all relational constraints $(C, T)$ relaxing $(R, S)$ and verifying $|C| \leq s$, only those are required to be in $Q$ that are indeed relation pairs. Then we get

$$
Q \cup \bigcup_{m \in \mathbb{N}_{+}}\left\{(R, S) \in \operatorname{Rp}_{A}^{(m)}|\forall C \subseteq R,| C \mid \leq s \forall C \supseteq T \supseteq S:(C, T) \in Q\right\} .
$$

This set still differs from $(s+1)-\mathrm{LOC}_{A} Q$ as defined above. For instance, for any $s \geq 1$ and $Q=\emptyset$, we have $(s+1)-\mathrm{LOC}_{A} Q=\emptyset$, while the previously displayed collection contains all relation pairs $(R, S) \in \operatorname{Rp}_{A}$ where $|S|>s$.

We do not see an obvious way how to turn $\mathbf{L O}_{s}$ into $(s+1)-\mathrm{LOC}_{A}$. 


\section{Semiclones and the full iterative Post algebra}

The following definition is very similar to that of a clone of operations. The only difference is that a clone $F \subseteq \mathrm{O}_{A}$ is additionally required to contain the set $\mathrm{J}_{A}$ of projections as a subset.

Definition 3.1. A (concrete) semiclone (of operations) on a set $A$ is a subset $F \subseteq \mathrm{O}_{A}$ of all finitary operations such that for all $m, n \in \mathbb{N}$, we have $f \circ\left(g_{0}, \ldots, g_{n-1}\right) \in F$ for each $f \in F^{(n)}$ and $\left(g_{0}, \ldots, g_{n-1}\right) \in\left(\left(F \cup \mathrm{J}_{A}\right)^{(m)}\right)^{n}$.

The closure property stated in Definition 3.1 is formulated in terms of partial composition operations on $\mathrm{O}_{A}$, as the functions making up the tupling all have to be of identical arity. However, it is possible to extend these operations in a conservative way to totally defined operations on $\mathrm{O}_{A}$ such that semiclones are exactly the subuniverses of a certain universal algebra on the carrier set $\mathrm{O}_{A}$ : for each $n, m \in \mathbb{N}$, each subset $I \subseteq n$, and any tuple $\left(g_{i}\right)_{i \in I} \in\left(\mathrm{J}_{A}^{(m)}\right)^{I}$ of $m$-ary projections, we define an $(|n \backslash I|+1)$-ary operation on $\mathrm{O}_{A}$, which maps $\left(f,\left(g_{i}\right)_{i \in n \backslash I}\right)$ to $f \circ\left(g_{i}\right)_{i \in n}$ provided that $f \in \mathrm{O}_{A}^{(n)}$ and $g_{i} \in \mathrm{O}_{A}^{(m)}$ for all $i \in n \backslash I$, and to $f$ otherwise. If we collect all the finitary operations obtained in this way in a set $\Phi \subseteq \mathrm{O}_{\mathrm{O}_{A}}$, then it becomes clear that $F \subseteq \mathrm{O}_{A}$ is a semiclone if and only if it is a subuniverse of the algebra $\left\langle\mathrm{O}_{A} ; \Phi\right\rangle$.

Hence, the set $\mathcal{S}_{A}:=\operatorname{Sub}\left(\left\langle\mathrm{O}_{A} ; \Phi\right\rangle\right)$ of all semiclones on $A$ bears the structure of a complete algebraic lattice with respect to set-inclusion, and is, in particular, a closure system. The corresponding closure operator will be denoted by []$_{\mathrm{O}_{A}}$.

Evident, trivial examples of semiclones are the empty set of operations and any clone $F \subseteq \mathrm{O}_{A}$. Moreover, we have the following class of examples.

Lemma 3.2. For a set $G \subseteq \mathrm{O}_{A}^{(1)}$ of unary transformations, abbreviate its generated transformation semigroup by $S:=\langle G\rangle_{\left\langle\mathrm{O}_{A}^{(1)} ; \circ\right\rangle}$. Then we have

$$
[G]_{\mathrm{O}_{A}}=\left\{f \circ e_{i}^{(n)} \mid i \in n \wedge n \in \mathbb{N}_{+} \wedge f \in S\right\} .
$$

Corollary 3.3. The unary parts of semiclones $\left\{F^{(1)} \mid F \in \mathcal{S}_{A}\right\}$ are precisely all (carrier sets of) transformation semigroups on $A$.

As mentioned in the introduction, semiclones are not a new invention. They are just the subuniverses ("closed classes of functions") of the full iterative Post algebra. In order to see this, we need a few definitions.

For $n \in \mathbb{N}_{+}$, define $\alpha_{n}^{\zeta}: n \rightarrow n$ by $\alpha_{n}^{\zeta}(i):=i+1(\bmod n)$ and $\alpha_{0}^{\zeta}:=\operatorname{id}_{0}$. Moreover, let $\alpha_{n}^{\tau}: n \rightarrow n$ be the transposition $(0,1)$ for $n \in \mathbb{N}$ with $n \geq 2$, and put $\alpha_{n}^{\tau}:=\operatorname{id}_{n}$ for $n \in\{0,1\}$. We continue by defining $\alpha_{n}^{\Delta}: n \rightarrow n-1$ via $\alpha_{n}^{\Delta}(i):=\max (0, i-1)$ for $n \in \mathbb{N}$ with $n \geq 2$, letting $\alpha_{n}^{\Delta}:=\operatorname{id}_{n}$ for $n \in\{0,1\}$ and declaring the map $\alpha_{n}^{\nabla}: n \rightarrow n+1$ by $\alpha_{n}^{\nabla}(i):=i+1$ for any $n \in \mathbb{N}$. 
On this basis, we define for $\omega \in\{\zeta, \tau, \Delta, \nabla\}$ a unary map $\omega: \mathrm{O}_{A} \rightarrow \mathrm{O}_{A}$ by $\omega(f):=\delta_{\alpha_{\operatorname{ar}(f)}^{\omega}}(f)$ for $f \in \mathrm{O}_{A}$. Moreover, for $f, g \in \mathrm{O}_{A}, n:=\operatorname{ar}(f)$ and $m:=\operatorname{ar}(g)$, we construct $f * g \in \mathrm{O}_{A}^{(k)}$, where $k:=\max (0, n+m-1)$, as follows: if $n \geq 2$, we put $f * g:=f \circ\left(g \circ\left(e_{i}^{(k)}\right)_{i \in m},\left(e_{m+j}^{(k)}\right)_{j \in n-1}\right)$; for $n=1$, we define the product $f * g:=f \circ g \circ\left(e_{i}^{(k)}\right)_{i \in m}$ whenever $m>0$, and $f * g:=f \circ g$ if $m=0$; for $n=0$, we define $f * g:=f$ in case that $k=0$, and $f * g:=f \circ\left(e_{i}^{(k)}\right)_{i \in 0}$ otherwise (where $\left(e_{i}^{(k)}\right)_{i \in 0}$ by definition is the unique map from $A^{k}$ to $A^{0}$ ).

In this way, we obtain an algebra $\mathrm{O}_{\mathbf{A}}:=\left\langle\mathrm{O}_{A} ; \zeta, \tau, \Delta, \nabla, *\right\rangle$ of arity type $(1,1,1,1,2)$ that we call full iterative Post algebra. It is easy to see that $\mathrm{O}_{A} \backslash \mathrm{O}_{A}^{(0)}$ is a subuniverse, and the corresponding subalgebra is the one that has been introduced under precisely the same name in [27]. The difference in terminology is just of a technical nature and shows up because we wish to accommodate all nullary constants in our framework.

The algebra $\mathrm{O}_{\mathbf{A}}$ obviously is less prodigal of its fundamental operations than $\left\langle\mathrm{O}_{A} ; \Phi\right\rangle$ above. The following lemma proves that both do the same job.

Lemma 3.4. The semiclones on $A$ are exactly the subuniverses of the full iterative Post algebra: $\mathcal{S}_{A}=\operatorname{Sub}\left(\mathrm{O}_{\mathbf{A}}\right)$.

The following facts regarding the relationship of semiclones and clones are well known (see [17, p. 5 et seq.] or [18, p. 8 et seq.], Lemmata 3 and 4, and Satz 1). In this context, we recollect that $\langle F\rangle_{\mathrm{O}_{A}}$ denotes the least clone containing some set $F \subseteq \mathrm{O}_{A}$, i.e., the clone generated by $F$. The symbol $\mathcal{L}_{A}$ stands for the set of all clones on $A$.

Lemma 3.5. For any set $F \subseteq \mathrm{O}_{A}$ and any $0 \leq i<n, n \in \mathbb{N}$, the following assertions are true:

(a) $\left[\left\{e_{i}^{(n)}\right\}\right]_{\mathrm{O}_{A}}=\mathrm{J}_{A}$.

(b) $\left[F \cup\left\{e_{i}^{(n)}\right\}\right]_{\mathrm{O}_{A}}=[F]_{\mathrm{O}_{A}} \cup \mathrm{J}_{A}=\langle F\rangle_{\mathrm{O}_{A}}$.

(c) $F \cap \mathrm{J}_{A} \in\left\{\emptyset, \mathrm{J}_{A}\right\}$ for $F \in \mathcal{S}_{A}$.

(d) $\mathcal{L}_{A}=\left\{G \in \mathcal{S}_{A} \mid G \cap \mathrm{J}_{A} \neq \emptyset\right\}$.

As a consequence of the previous lemma, we can describe those semiclones whose unary parts yield proper transformation semigroups.

Corollary 3.6. On any set $A$, we have

$$
\left\{F^{(1)} \mid F \in \mathcal{S}_{A} \backslash \mathcal{L}_{A}\right\}=\left\{S \subseteq \mathrm{O}_{A}^{(1)} \backslash\left\{\mathrm{id}_{A}\right\} \mid(S, \circ) \text { is a semigroup }\right\} .
$$

The Galois correspondence Polp - Invp gives us plenty of examples of semiclones (cf. [19, Lemma 2, p. 12] for the situation without nullary operations).

Lemma 3.7. Any polymorphism set $\operatorname{Polp}_{A} Q$ with $Q \subseteq \mathrm{Rp}_{A}$ is a semiclone. 
Corollary 3.8. For any set $F \subseteq \mathrm{O}_{A}$, we have $[F]_{\mathrm{O}_{A}} \subseteq \operatorname{Polp}_{A} \operatorname{Invp}_{A} F$ and $\operatorname{Invp}_{A} F=\operatorname{Invp}_{A}[F]_{\mathrm{O}_{A}}$.

The next lemma (cf. [19, Lemma 3, p. 13]) clarifies which sets of relation pairs yield proper clones.

Lemma 3.9. For $Q \subseteq \mathrm{Rp}_{A}$, a semiclone $\operatorname{Polp}_{A} Q$ is a clone if and only if $\varrho=\varrho^{\prime}$ holds for all $\left(\varrho, \varrho^{\prime}\right) \in Q$.

Note that (along with an appropriate generalisation of preservation) the three previous statements remain true if one considers relation pairs of arbitrary, possibly infinite arity, i.e., pairs $(R, S)$, where $S \subseteq R \subseteq A^{K}$ for sets $K$.

The following result is in analogy to [30, Proposition 1.11(a),(b), p. 17].

Lemma 3.10. For $0<s \leq \aleph_{0}$ and any set $Q \subseteq \mathrm{Rp}_{A}^{(<s)}$ of less than s-ary relation pairs, we have $s-\operatorname{Loc}_{A} \operatorname{Polp}_{A} Q=\operatorname{Polp}_{A} Q$.

\section{Relation pair clones}

In this section, we first recall the so-called general superposition of relations ([30, Definition 3.4(R4), p. 27], see also [29, Definition 2.2(ii), p. 258] and [3]), which comes into play when generalising the notion of relational clone from finite carrier sets to arbitrary ones. It is not surprising that it will be important for the generalisation of relation pair algebras as introduced in [17, p. 21] (see also [19, p. 16]) to carrier sets of arbitrary cardinality, as well.

Definition 4.1. Let $A$ be any carrier set, moreover let index sets $I$ and $\mu$ (one could in principle restrict to ordinal numbers, but this only makes working with the definition more technical), natural numbers $m, m_{i} \in \mathbb{N}$ (for $i \in I$ ), mappings $\left(\alpha_{i}: m_{i} \rightarrow \mu\right)_{i \in I}$ and $\beta: m \rightarrow \mu$, and relations $\varrho_{i} \in \mathrm{R}_{A}^{\left(m_{i}\right)}($ for $i \in I$ ) be given. The general superposition of these relations with respect to the given data is defined to be the $m$-ary relation

$$
\begin{aligned}
\bigwedge_{\left(\alpha_{i}\right)_{i \in I}}^{\beta}\left(\varrho_{i}\right)_{i \in I}: & =\left\{y \in A^{m} \mid \exists a \in A^{\mu}: y=a \circ \beta \wedge \forall i \in I: a \circ \alpha_{i} \in \varrho_{i}\right\} \\
& =\left\{a \circ \beta \mid a \in A^{\mu} \wedge \forall i \in I: a \circ \alpha_{i} \in \varrho_{i}\right\} .
\end{aligned}
$$

We mention in passing that, in general, a relational clone can be defined as any set $Q \subseteq \mathrm{R}_{A}$ that is closed with respect to general superposition. That is, whenever data as in Definition 4.1 is given and all relations $\varrho_{i}$, for $i \in I$, belong to $Q$, then also $\bigwedge_{\left(\alpha_{i}\right)_{i \in I}}^{\beta}\left(\varrho_{i}\right)_{i \in I}$ has to be an element of $Q$ (if nullary relations are disregarded, then one restricts the integers $m$ and $\left(m_{i}\right)_{i \in I}$ to positive ones only). Depending on the set $A$, one can work out cardinality bounds on the sets $I$ and $\mu$ involved in this closure property, but this is not our concern here. 
Definition 4.2. Let $A$ be any carrier set, $I, \mu, m, m_{i}, \alpha_{i}: m_{i} \rightarrow \mu$ for $i \in I$, and $\beta: m \rightarrow \mu$ as in Definition 4.1. For relation pairs $\left(\varrho_{i}, \varrho_{i}^{\prime}\right) \in \operatorname{Rp}_{A}^{\left(m_{i}\right)}$, for $i \in I$, we define their general superposition to be

$$
\bigwedge_{\left(\alpha_{i}\right)_{i \in I}}^{\beta}\left(\varrho_{i}, \varrho_{i}^{\prime}\right)_{i \in I}:=\left(\bigwedge_{\left(\alpha_{i}\right)_{i \in I}}^{\beta}\left(\varrho_{i}\right)_{i \in I}, \bigwedge_{\left(\alpha_{i}\right)_{i \in I}}^{\beta}\left(\varrho_{i}^{\prime}\right)_{i \in I}\right) .
$$

It is easy to see that this definition is well-defined, i.e., that we really have $\bigwedge_{\left(\alpha_{i}\right)_{i \in I}}^{\beta}\left(\varrho_{i}, \varrho_{i}^{\prime}\right)_{i \in I} \in \operatorname{Rp}_{A}^{(m)}$ in the situation described in Definition 4.2. This allows us to define relation pair clones as such sets of relation pairs that are closed under general superposition.

Definition 4.3. We say that for some carrier $A$, a set $Q \subseteq \mathrm{Rp}_{A}$ is a relation pair clone if and only if the following condition is satisfied: whenever $I, \mu$, $m, m_{i}, \alpha_{i}: m_{i} \rightarrow \mu$ for $i \in I$, and $\beta: m \rightarrow \mu$ are as in Definition 4.1, and $\left(\varrho_{i}, \varrho_{i}^{\prime}\right) \in Q^{\left(m_{i}\right)}$ are given for $i \in I$, then also $\bigwedge_{\left(\alpha_{i}\right)_{i \in I}}^{\beta}\left(\varrho_{i}, \varrho_{i}^{\prime}\right)_{i \in I} \in Q^{(m)}$.

One can routinely check that for a given carrier set $A$, the collection of all relation pair clones on $A$ is a closure system. We denote the corresponding closure operator by $Q \mapsto[Q]_{\mathrm{Rp}_{A}}$ for $Q \subseteq \mathrm{Rp}_{A}$ and refer to $[Q]_{\mathrm{Rp}_{A}}$ as the relation pair clone generated by $Q$.

Note that for finite carrier sets $A \neq \emptyset$, and provided that $(\emptyset, \emptyset) \in Q^{(m)}$ for all $m \in \mathbb{N}$, our concept of locally closed relation pair clone, by taking $Q \backslash \operatorname{Rp}_{A}^{(0)}$, subsumes that of subuniverses of the full relation pair algebra defined in [17, p. 21] (see also [19, p. 16]).

There are two issues here: the necessity to add local closure and the requirement that pairs of empty relations have to belong to relation pair algebras in Harnau's sense. We noted above in Corollary 2.12 that for finite carrier sets, closure under relaxation coincides with our local closure of relation pairs. Moreover, we shall prove in Theorem 5.9 that the closed sets with respect to $\operatorname{Invp}_{A} \operatorname{Polp}_{A}$ are precisely the locally closed relation pair clones, which implies for finite carrier sets that they are exactly those relation pair clones that are closed with respect to relaxations. In [17] and [19], this additional closure property (with the goal of characterising the Galois closures) has been incorporated into the definition of the full relation pair algebra via multioperations $d_{\mathrm{v}}$ and $d_{\mathrm{h}}$; however, it has been noted that these operators are of a different nature than the other fundamental operations of relation pair algebras. Comparing to the situation known from clones and relational clones on arbitrary domains (see [30, 29, 34]) and looking from the perspective of infinite carrier sets, which requires local closures anyway, it is justified to modify Harnau's definition by separating closure properties related to concrete constructions involving relations from local interpolation properties. We mention that for finite $A$, the constructive part can be expressed via interpretations of primitive positive formulæ in both components. In fact, it was noted by Ágnes Szendrei that given a set $Q \subseteq \mathrm{Rp}_{A}$, one may consider the relational 
structures $\underset{\sim}{\mathbf{A}}=\left\langle A ;(\varrho)_{\left(\varrho, \varrho^{\prime}\right) \in Q}\right\rangle$ and $\underset{\mathbf{A}^{\prime}}{\mathbf{A}^{\prime}}=\left\langle A ;\left(\varrho^{\prime}\right)_{\left(\varrho, \varrho^{\prime}\right) \in Q}\right\rangle$ and primitive positively definable relations on the product $\underset{\sim}{\mathbf{A}} \times \underbrace{\mathbf{A}^{\prime}}$ : if $\varphi$ is a primitive positive formula in the language of $Q$ (including equality) with at most $m$ free variables, then it defines the following $m$-ary relation on the product:

$$
\begin{aligned}
\hat{\sigma} & :=\left\{\left(\left(x_{i}, y_{i}\right)\right)_{i \in m} \in\left(A^{2}\right)^{m} \mid(\underbrace{\mathbf{A}}_{\sim} \times \underbrace{\mathbf{A}^{\prime}}_{\sim},\left(\left(x_{i}, y_{i}\right)\right)_{i \in m}) \models \varphi\right\} \\
& =\left\{\left(\left(x_{i}, y_{i}\right)\right)_{i \in m} \in\left(A^{2}\right)^{m} \mid(\underbrace{\mathbf{A}}_{i}\left(x_{i}\right)_{i \in m}) \models \varphi \wedge(\underbrace{\mathbf{A}^{\prime}}_{\sim},\left(y_{i}\right)_{i \in m}) \models \varphi\right\} \\
& =\left\{\left(\left(x_{i}, y_{i}\right)\right)_{i \in m} \in\left(A^{2}\right)^{m} \mid\left(\left(x_{i}\right)_{i \in m},\left(y_{i}\right)_{i \in m}\right) \in \sigma \times \sigma^{\prime}\right\},
\end{aligned}
$$

where $\sigma:=\left\{\mathbf{x} \in A^{m} \mid(\underset{\mathbf{A}}{\mathbf{x}} \mathbf{x}) \models \varphi\right\}$ and $\sigma^{\prime}:=\left\{\mathbf{x} \in A^{m} \mid(\underbrace{\mathbf{A}^{\prime}}, \mathbf{x}) \models \varphi\right\}$. If $\sigma$ and $\sigma^{\prime}$ are both non-empty, then one may obtain the relation pair $\left(\sigma, \sigma^{\prime}\right)$ defined by $\varphi$ as projections of $\hat{\sigma}$. If one of them is the empty set, then $\hat{\sigma}=\emptyset$ and therefore both projections will be empty. Thus, only taking projections of $\hat{\sigma}$ (i.e., of pp-definable relations in the product $\underset{\sim}{\mathbf{A}} \times \underbrace{\mathbf{A}^{\prime}}$ ) will never produce relation pairs $\left(\sigma, \sigma^{\prime}\right)$ where $\sigma^{\prime}=\emptyset \subsetneq \sigma$, which is certainly needed, e.g., to model intersection in both components. However, collecting all pairs $\left(\sigma, \sigma^{\prime}\right)$ arising from primitive positive formulæ $\varphi$ correctly describes the closure $[Q]_{\mathrm{Rp}_{A}}$ in the case of finite carrier sets.

The second issue pointed out above is related to nullary operations. In the literature, these are often neglected, which makes it necessary for relation pair algebras ([19]) and for relational clones (relation algebras, [30]) to contain the empty pair $(\emptyset, \emptyset)$ and the empty relation, respectively, in order to be in accordance with the corresponding Galois theory.

If nullary operations are given their proper place, this absurdity vanishes (see [3] for clones and relational clones); then empty relations (pairs) get a true function, indicating by their presence the absence of nullary operations on the dual side (see Lemma 4.8 below). This is also the reason why we cannot and do not add the empty pairs of all arities as nullary constants to the closure condition of relation pair clones.

Relational clones (as given in [3, Definition 2.2, p. 8]) relate to relation pair clones in the following way:

Lemma 4.4. For any carrier set $A$, a subset $Q \subseteq \mathrm{R}_{A}$ is a relational clone if and only if $P:=\biguplus_{m \in \mathbb{N}}\left\{(\varrho, \varrho) \mid \varrho \in Q^{(m)}\right\}$ is a relation pair clone.

Lemma 4.5. Whenever $Q \subseteq \mathrm{Rp}_{A}$ is a relation pair clone on some set $A$, then

$$
\begin{aligned}
Q^{\prime} & :=\left\{\varrho \mid\left(\varrho, \varrho^{\prime}\right) \in Q^{(m)} \text { for some } m \in \mathbb{N}\right\}, \\
Q^{\prime \prime} & :=\left\{\varrho^{\prime} \mid\left(\varrho, \varrho^{\prime}\right) \in Q^{(m)} \text { for some } m \in \mathbb{N}\right\} \text { and } \\
Q^{\prime \prime \prime} & :=\left\{\varrho \mid(\varrho, \varrho) \in Q^{(m)} \text { for some } m \in \mathbb{N}\right\}
\end{aligned}
$$

are relational clones on $A$. 
Similarly as for semiclones, the Galois correspondence Polp-Invp provides many examples of relation pair clones (see [17, Lemma 9, p. 21] or [19, Lemma 9, p. 16] for the case of finite carrier sets; cf. [13, Lemma 3.1, p. 154] for the general framework of relational constraints and conjunctive minors).

Lemma 4.6. For each $F \subseteq \mathrm{O}_{A}$ the set $\operatorname{Invp}_{A} F$ is a relation pair clone.

Corollary 4.7. For any set $Q \subseteq \mathrm{Rp}_{A}$, we have $[Q]_{\mathrm{Rp}_{A}} \subseteq \operatorname{Invp}_{A} \operatorname{Polp}_{A} Q$ and $\operatorname{Polp}_{A} Q=\operatorname{Polp}_{A}[Q]_{\mathrm{Rp}_{A}}$.

Next, we address how nullary operations affect relation pair algebras.

Lemma 4.8. For $F \subseteq \mathrm{O}_{A}$, we have $(\emptyset, \emptyset) \in \operatorname{Invp}_{A} F$ if and only if we have $F \subseteq \mathrm{O}_{A} \backslash \mathrm{O}_{A}^{(0)}$.

The following result is in analogy to [30, Proposition 1.11(a'),(b'), p. 17].

Lemma 4.9. For $0<s \leq \aleph_{0}$ and any set $F \subseteq \mathrm{O}_{A}^{(<s)}$ of less than s-ary operations, we have $s-\mathrm{LOC}_{A} \operatorname{Invp}_{A} F=\operatorname{Invp}_{A} F$.

\section{Characterisation of closures related to Polp-Invp}

In this section, we characterise, for any cardinal $0<s \leq \aleph_{0}$, the operators $\operatorname{Polp}_{A} \operatorname{Invp}_{A}^{(<s)}$ and $\operatorname{Invp}_{A} \operatorname{Polp}_{A}^{(<s)}$ as $s$-local closures of the generated semiclone and relation pair clone, respectively. Subsequently, we present a few consequences of these theorems.

5.1. The operational side. For our task, it is helpful to gather some knowledge about the least (with respect to $\leq$ and thus a least among several equivalent ones with respect to $\preceq$ ) pair $\left(\varrho, \varrho^{\prime}\right) \in \mathrm{Rp}_{A}^{(m)}$ being invariant for some set $F \subseteq \mathrm{O}_{A}$ and satisfying $B \subseteq \varrho$ for a given finite set $B \subseteq \mathrm{R}_{A}^{(m)}$, for $m \in \mathbb{N}$. Addressing this issue, the following lemma generalises Proposition 2.4 of [30, p. 21].

Lemma 5.1. Let $F \subseteq \mathrm{O}_{A}$ be a set of operations and $\mathbf{b} \in\left(A^{m}\right)^{n}$ for some $m, n \in \mathbb{N}$; set $B:=\{\mathbf{b}(j) \mid 0 \leq j<n\} \subseteq A^{m}$. Then the pair $\Gamma_{F}(B):=\left(\varrho, \varrho^{\prime}\right)$, where $\varrho:=\left\{f \circ(\mathbf{b}) \mid f \in\langle F\rangle_{\mathrm{O}_{A}}^{(n)}\right\}$ and $\varrho^{\prime}:=\left\{f \circ(\mathbf{b}) \mid f \in[F]_{\mathrm{O}_{A}}^{(n)}\right\}$, is the least pair (with respect to $\leq$ ) in $\operatorname{Invp}_{A}^{(m)} F$ satisfying $B \subseteq \varrho$.

Note that the lemma also shows that the relations $\varrho, \varrho^{\prime} \in \mathrm{R}_{A}^{(m)}$ do not depend on the order of the entries of the tuple $\mathbf{b}$. Furthermore, instead of the finite cardinal $m$, any cardinal or, in fact, any indexing set $K$ can be used, provided the notion of preservation is straightforwardly extended to relation pairs of arbitrary arity, i.e., pairs $(R, S)$ such that $S \subseteq R \subseteq A^{K}$.

Proof. First of all, as $\mathrm{J}_{A}^{(n)} \subseteq\langle F\rangle_{\mathrm{O}_{A}}^{(n)}$, we have $B \subseteq \varrho$. Next, we prove that $\left(\varrho, \varrho^{\prime}\right) \in \operatorname{Invp}_{A} F$; let $\ell \in \mathbb{N}, g \in F^{(\ell)}$, and $\mathbf{r}=\left(r_{j}\right)_{0 \leq j<\ell} \in \varrho^{\ell}$. By construction 
of $\varrho$, for each $0 \leq j<\ell$, there exists some $f_{j} \in\langle F\rangle_{\mathrm{O}_{A}}^{(n)}=[F]_{\mathrm{O}_{A}}^{(n)} \cup \mathrm{J}_{A}^{(n)}$ (see Lemma 3.5(b)) such that $r_{j}=f_{j} \circ(\mathbf{b})$. Using Lemma 2.1(c), we have

$$
g \circ(\mathbf{r})=g \circ\left(f_{0} \circ(\mathbf{b}), \ldots, f_{\ell-1} \circ(\mathbf{b})\right)=\left(g \circ\left(f_{0}, \ldots, f_{\ell-1}\right)\right) \circ(\mathbf{b}) \in \varrho^{\prime},
$$

since $g \circ\left(f_{0}, \ldots, f_{\ell-1}\right) \in[F]_{\mathrm{O}_{A}}^{(n)}$ by the closure property of semiclones.

Finally, we prove that any pair $\left(\sigma, \sigma^{\prime}\right) \in \operatorname{Invp}_{A}^{(m)} F$ satisfying $B \subseteq \sigma$ fulfils $\left(\varrho, \varrho^{\prime}\right) \leq\left(\sigma, \sigma^{\prime}\right)$. By Corollary 3.8, we know $\left(\sigma, \sigma^{\prime}\right) \in \operatorname{Invp}_{A} F=\operatorname{Invp}_{A}[F]_{\mathrm{O}_{A}}$, so since $B \subseteq \sigma$, we have $f \circ(\mathbf{b}) \in \sigma^{\prime}$ for any $f \in[F]_{\mathrm{O}_{A}}^{(n)}$. Therefore, $\varrho^{\prime} \subseteq \sigma^{\prime} \subseteq \sigma$. As, by Lemma 3.5(b), $\langle F\rangle_{\mathrm{O}_{A}}^{(n)}=[F]_{\mathrm{O}_{A}}^{(n)} \cup \mathrm{J}_{A}^{(n)}$, it follows that $\varrho=\varrho^{\prime} \cup B$. We have $B \subseteq \sigma$ and $\varrho^{\prime} \subseteq \sigma$, so $\varrho \subseteq \sigma$. Hence, $\left(\varrho, \varrho^{\prime}\right) \leq\left(\sigma, \sigma^{\prime}\right)$.

Corollary 5.2. Let $F \subseteq \mathrm{O}_{A}, n \in \mathbb{N}$, and $X \subseteq A^{n}$ be any subset of finite cardinality $|X|=: k<\aleph_{0}$; moreover, consider an arbitrary bijection $\beta: k \rightarrow X$ as fixed. Defining $B:=\left\{\left.e_{i}^{(n)}\right|_{X} \circ \beta \mid 0 \leq i<n\right\} \subseteq A^{k}$, as well as $k$-ary relations $\varrho_{X, n}:=\left\{\left.f\right|_{X} \circ \beta \mid f \in\langle F\rangle_{\mathrm{O}_{A}}^{(n)}\right\}$ and $\varrho_{X, n}^{\prime}:=\left\{\left.f\right|_{X} \circ \beta \mid f \in[F]_{\mathrm{O}_{A}}^{(n)}\right\}$, we have $\left(\varrho_{X, n}, \varrho_{X, n}^{\prime}\right)=\Gamma_{F}(B) \in \operatorname{Invp}_{A}^{(k)} F$.

Theorem 5.3. For $0<s \leq \aleph_{0}$ and any set of operations $F \subseteq \mathrm{O}_{A}$, we have the equality $\operatorname{Polp}_{A} \operatorname{Invp}_{A}^{(<s)} F=s-\operatorname{Loc}_{A}[F]_{\mathrm{O}_{A}}$.

Proof. We have $[F]_{\mathrm{O}_{A}} \subseteq \operatorname{Polp}_{A} \operatorname{Invp}_{A}^{(<s)}[F]_{\mathrm{O}_{A}}=\operatorname{Polp}_{A} \operatorname{Invp}_{A}^{(<s)} F$ by Corollary 3.8, whence $s-\operatorname{Loc}_{A}[F]_{O_{A}} \subseteq s-\operatorname{Loc}_{A} \operatorname{Polp}_{A} \operatorname{Invp}_{A}^{(<s)} F=\operatorname{Polp}_{A} \operatorname{Invp}_{A}^{(<s)} F$, using Lemma 3.10.

For the converse inclusion, take $g \in \operatorname{Polp}_{A}^{(n)} \operatorname{Inv}_{A}^{(<s)} F$ for any $n \in \mathbb{N}$; we want to prove that $g \in s-\operatorname{Loc}_{A}^{(n)}[F]_{\mathrm{O}_{A}}$. To do so, we consider any finite $X \subseteq A^{n}$ where $k:=|X|<s$ and an arbitrary bijection $\beta: k \rightarrow X$. Now Corollary 5.2 yields that $\left(\varrho_{X, n}, \varrho_{X, n}^{\prime}\right) \in \operatorname{Invp}_{A}^{(k)} F \subseteq \operatorname{Invp}_{A}^{(<s)} F$, wherefore $g \triangleright\left(\varrho_{X, n}, \varrho_{X, n}^{\prime}\right)$. Moreover, we have $B=\left\{\left.e_{i}^{(n)}\right|_{X} \circ \beta \mid 0 \leq i<n\right\} \subseteq \varrho_{X, n}$, whence we obtain $\left.g\right|_{X} \circ \beta=\left.g \circ\left(e_{i}^{(n)}\right)_{0 \leq i<n}\right|_{X} \circ \beta=g \circ\left(\left.e_{i}^{(n)}\right|_{X} \circ \beta\right)_{0 \leq i<n} \in \varrho_{X, n}^{\prime}$. Thus, by definition of $\varrho_{X, n}^{\prime}$, there has to exist some $f \in[F]_{\mathrm{O}_{A}}^{(n)}$ such that $\left.g\right|_{X} \circ \beta=\left.f\right|_{X} \circ \beta$, implying $\left.g\right|_{X}=\left.f\right|_{X}$ by bijectivity of $\beta$. This proves $g \in s-\operatorname{Loc}_{A}^{(n)}[F]_{\mathrm{O}_{A}}$.

Lemma 5.4. Any relation pair clone $Q \subseteq \mathrm{Rp}_{A}$ on a non-empty carrier set $A$ satisfies $Q^{(m)} \subseteq\left[Q^{(s)}\right]_{\mathrm{Rp}_{A}}$ for all $m, s \in \mathbb{N}$ with $m \leq s$.

For $A=\emptyset$ and $s \in \mathbb{N}$, we have $Q^{(s)} \subseteq\left[Q^{(0)}\right]_{\mathrm{Rp}_{A}}$ for a relation pair clone $Q$.

Corollary 5.5. For $s \in \mathbb{N}$ and any set of operations $F \subseteq \mathrm{O}_{A}$ on $A \neq \emptyset$, we have the equality $\operatorname{Polp}_{A} \operatorname{Invp}_{A}^{(s)} F=(s+1)-\operatorname{Loc}_{A}[F]_{\mathrm{O}_{A}}$.

If $A=\emptyset$, we have $s-\operatorname{Loc}_{A}[F]_{\mathrm{O}_{A}}=\operatorname{Polp}_{A} \operatorname{Invp}_{A}^{(0)} F=1-\operatorname{Loc}_{A}[F]_{\mathrm{O}_{A}}$ for any $F \subseteq \mathrm{O}_{A}$ and $0<s \leq \aleph_{0}$.

The second corollary proves that a set $F \subseteq \mathrm{O}_{A}$ is closed with respect to []$_{\mathrm{O}_{A}}$ and $s-\mathrm{Loc}_{A}$ if (and clearly only if) it is closed with respect to the operator 
$s-\operatorname{Loc}_{A}[]_{\mathrm{O}_{A}}$. This fact can be seen as a generalisation of Lemma 2.5(ii),(iii) in [30, p. 22], where similar results have been proven for clones.

Corollary 5.6. For $0<s \leq \aleph_{0}$ a set $F \subseteq \mathrm{O}_{A}$ of operations is an s-locally closed semiclone if and only if $s-\operatorname{Loc}_{A}[F]_{\mathrm{O}_{A}}=F$.

Corollary 5.7 (cf. Lemma 2.6 in [30, p. 22]). For every $F \subseteq \mathrm{O}_{A}$, we have $\operatorname{Invp}_{A}^{(<m)} F=\operatorname{Invp}_{A}^{(<m)}[F]_{\mathrm{O}_{A}}=\operatorname{Invp}_{A}^{(<m)} s-\operatorname{Loc}_{A}[F]_{\mathrm{O}_{A}}$ for $0<m \leq s \leq \aleph_{0}$.

5.2. The side of relation pairs. We start by preparing the proof of our theorem with a lemma.

Lemma 5.8. Let $Q \subseteq \mathrm{Rp}_{A}$ be any set of relation pairs, $m \in \mathbb{N}$ an arity, and $B \subseteq A^{m}$ a finite subset of cardinality $n:=|B|$. Consider any enumeration $\mathbf{b}=\left(b_{0}, \ldots, b_{s-1}\right) \in B^{s}$ of $B=\left\{b_{0}, \ldots, b_{s-1}\right\}$ (for $\left.s \geq n\right)$ and define

$$
\mu_{B}^{\prime}:=\left\{f \circ(\mathbf{b}) \mid f \in \operatorname{Polp}_{A}^{(s)} Q\right\}, \quad \mu_{B}:=\left\{f \circ(\mathbf{b}) \mid f \in \operatorname{Pol}_{A}^{(s)} Q_{1}\right\},
$$

where $Q_{1}:=\left\{\varrho \in \mathrm{R}_{A} \mid\left(\varrho, \varrho^{\prime}\right) \in Q\right\}$.

(a) The pair $\left(\mu_{B}, \mu_{B}^{\prime}\right)$ can be obtained from $Q$ by general superpositions, i.e., $\left(\mu_{B}, \mu_{B}^{\prime}\right) \in[Q]_{\mathrm{Rp}_{A}}$.

(b) For $F:=\operatorname{Polp}_{A} Q$, one may obtain $\Gamma_{F}(B)$ as a relaxation of $\left(\mu_{B}, \mu_{B}^{\prime}\right)$, that is, $\Gamma_{F}(B) \in \rightarrow[Q]_{\mathrm{Rp}_{A}} \leftarrow$.

Proof. (a): In order to prove that $\left(\mu_{B}, \mu_{B}^{\prime}\right) \in[Q]_{\mathrm{Rp}_{A}}$, we shall exhibit a general composition producing this relation pair from the ones in $Q$. Using the notation from Definition 4.2, we choose $\mu:=A^{s}$ and define $\beta: m \rightarrow A^{s}$ by $\beta(i):=\left(b_{0}(i), \ldots, b_{s-1}(i)\right)$ for $0 \leq i<m$. Moreover, for $n \in \mathbb{N}$ and $\left(\varrho, \varrho^{\prime}\right) \in Q^{(n)}$, we put $I_{n,\left(\varrho, \varrho^{\prime}\right)}:=\left\{\left(n, \varrho, \varrho^{\prime}, \mathbf{r}\right) \mid \mathbf{r} \in \varrho^{s}\right\}$; further, we define $I:=\biguplus_{n \in \mathbb{N}} \bigcup_{\left(\varrho, \varrho^{\prime}\right) \in Q^{(n)}} I_{n,\left(\varrho, \varrho^{\prime}\right)}$. Finally, for $\left(n, \varrho, \varrho^{\prime}, \mathbf{r}\right) \in I$, let the function $\alpha_{n, \varrho, \varrho^{\prime}, \mathbf{r}}: n \rightarrow A^{s}$ be given by $\alpha_{n, \varrho, \varrho^{\prime}, \mathbf{r}}(j):=\left(r_{0}(j), \ldots, r_{s-1}(j)\right)$ for $0 \leq j<n$, where $\mathbf{r}=\left(r_{0}, \ldots, r_{s-1}\right) \in \varrho^{s}$.

We claim now that $\left(\mu_{B}, \mu_{B}^{\prime}\right)=\bigwedge_{\left(\alpha_{n, \varrho, \varrho^{\prime}, \mathbf{r}}\right)_{\left(n, \varrho, \varrho^{\prime}, \mathbf{r}\right) \in I}^{\beta}}^{\beta}\left(\varrho, \varrho^{\prime}\right)_{\left(n, \varrho, \varrho^{\prime}, \mathbf{r}\right) \in I}$, which can be checked by the following calculation. For each $\left(n, \varrho, \varrho^{\prime}, \mathbf{r}\right) \in I$, denote by $\sigma_{n, \varrho, \varrho^{\prime}, \mathbf{r}}$ some relation in $\mathrm{R}_{A}^{(n)}$; we have

$$
\begin{aligned}
& \bigwedge^{\beta} \quad\left(\sigma_{n, \varrho, \varrho^{\prime}, \mathbf{r}}\right)_{\left(n, \varrho, \varrho^{\prime}, \mathbf{r}\right) \in I} \\
& \left(\alpha_{n, \varrho, \varrho^{\prime}, \mathbf{r}}\right)_{\left(n, \varrho, \varrho^{\prime}, \mathbf{r}\right) \in I} \\
& =\left\{\begin{array}{l|l}
(f(\beta(i)))_{0 \leq i<m} & \begin{array}{l}
f \in A^{A^{s}} \wedge \forall\left(n, \varrho, \varrho^{\prime}, \mathbf{r}\right) \in I: \\
\left(f\left(\alpha_{n, \varrho, \varrho^{\prime}, \mathbf{r}}(0)\right), \ldots, f\left(\alpha_{n, \varrho, \varrho^{\prime}, \mathbf{r}}(n-1)\right)\right) \in \sigma_{n, \varrho, \varrho^{\prime}, \mathbf{r}}
\end{array}
\end{array}\right\} \\
& =\left\{\begin{array}{l|r}
f \circ\left(b_{0}, \ldots, b_{s-1}\right) \mid \begin{array}{r}
f \in A^{s} \\
\wedge
\end{array} n \in \mathbb{N} \forall\left(\varrho, \varrho^{\prime}\right) \in Q^{(n)} \\
\forall \mathbf{r}=\left(r_{0}, \ldots, r_{s-1}\right) \in \varrho^{s}: \\
f \circ\left(r_{0}, \ldots, r_{s-1}\right) \in \sigma_{n, \varrho, \varrho^{\prime}, \mathbf{r}}
\end{array}\right\} \\
& =\left\{f \circ\left(b_{0}, \ldots, b_{s-1}\right) \mid f \in A^{A^{s}} \wedge \forall n \in \mathbb{N} \forall\left(\varrho, \varrho^{\prime}\right) \in Q^{(n)}: f \triangleright\left(\varrho, \sigma_{n, \varrho, \varrho^{\prime}, \mathbf{r}}\right)\right\} \text {. }
\end{aligned}
$$


Specialising this to $\sigma_{n, \varrho, \varrho^{\prime}, \mathbf{r}}:=\varrho^{\prime}$, we get

$$
\bigwedge_{\left(\alpha_{n, \varrho, \varrho^{\prime}, \mathbf{r}}\right)_{\left(n, \varrho, \varrho^{\prime}, \mathbf{r}\right) \in I}^{\beta}}^{\beta}\left(\varrho^{\prime}\right)_{\left(n, \varrho, \varrho^{\prime}, \mathbf{r}\right) \in I}=\left\{f \circ\left(b_{0}, \ldots, b_{s-1}\right) \mid f \in \operatorname{Polp}_{A}^{(s)} Q\right\}=\mu_{B}^{\prime} .
$$

Specialising once more to $\sigma_{n, \varrho, \varrho^{\prime}, \mathbf{r}}:=\varrho$, we obtain

$$
\bigwedge_{\left(\alpha_{n, \varrho, \varrho^{\prime}, \mathbf{r}}\right)_{\left(n, \varrho, \varrho^{\prime}, \mathbf{r}\right) \in I}}^{\beta}(\varrho)_{\left(n, \varrho, \varrho^{\prime}, \mathbf{r}\right) \in I}=\left\{f \circ\left(b_{0}, \ldots, b_{s-1}\right) \mid f \in \mathrm{Pol}_{A}^{(s)} Q_{1}\right\}=\mu_{B} .
$$

(b): By Lemma 3.7, we have $[F]_{\mathrm{O}_{A}}=F$, and therefore Lemma 3.5(b) yields $\langle F\rangle_{\mathrm{O}_{A}}=[F]_{\mathrm{O}_{A}} \cup \mathrm{J}_{A}=F \cup \mathrm{J}_{A}$. Hence, according to Lemma 5.1, we obtain that $\Gamma_{F}(B)=\left(\varrho, \varrho^{\prime}\right)$, wherein $\varrho=\left\{f \circ(\mathbf{b}) \mid f \in F^{(s)} \cup \mathrm{J}_{A}^{(s)}\right\}$ and $\varrho^{\prime}=\left\{f \circ(\mathbf{b}) \mid f \in F^{(s)}\right\}=\left\{f \circ(\mathbf{b}) \mid f \in \operatorname{Polp}_{A}^{(s)} Q\right\}=\mu_{B}^{\prime}$. Moreover, as obviously $F=\operatorname{Polp}_{A} Q \subseteq \operatorname{Pol}_{A} Q_{1}$, we have $\mu_{B}^{\prime}=\varrho^{\prime} \subseteq \varrho \subseteq \mu_{B}$. Since $\left(\mu_{B}, \mu_{B}^{\prime}\right) \in[Q]_{\mathrm{Rp}_{A}}$ by (a), we finally see $\Gamma_{F}(B)=\left(\varrho, \varrho^{\prime}\right) \in \rightarrow[Q]_{\mathrm{Rp}_{A}} \leftarrow$.

Theorem 5.9. For $0<s \leq \aleph_{0}$ and any set $Q \subseteq \mathrm{Rp}_{A}$ of relation pairs, we have $\operatorname{Invp}_{A} \operatorname{Polp}_{A}^{(<s)} Q=s-\mathrm{LOC}_{A}[Q]_{\mathrm{Rp}_{A}}$.

Proof. We have $[Q]_{\mathrm{Rp}_{A}} \subseteq \operatorname{Invp}_{A} \operatorname{Polp}_{A}^{(<s)}[Q]_{\mathrm{Rp}_{A}}=\operatorname{Invp}_{A} \operatorname{Polp}_{A}^{(<s)} Q$ by Corollary 4.7, so $s-\mathrm{LOC}_{A}[Q]_{\mathrm{Rp}_{A}} \subseteq s-\mathrm{LOC}_{A} \operatorname{Invp}_{A} \operatorname{Polp}_{A}^{(<s)} Q=\operatorname{Invp}_{A} \operatorname{Polp}_{A}^{(<s)} Q$ by Lemma 4.9 .

For the converse inclusion, let us consider $m \in \mathbb{N}$ and an arbitrary $m$-ary pair $\left(\sigma, \sigma^{\prime}\right) \in \operatorname{Invp}_{A}^{(m)} \operatorname{Polp}_{A}^{(<s)} Q$. To prove that $\left(\sigma, \sigma^{\prime}\right) \in s-\operatorname{LOC}_{A}[Q]_{\mathrm{Rp}_{A}}$, we take any subset $B \subseteq \sigma$ such that $|B|<s$. From Lemma 5.8(a), we get $\left(\mu_{B}, \mu_{B}^{\prime}\right) \in[Q]_{\mathrm{Rp}_{A}}$, and obviously we have $B \subseteq \mu_{B}$. Moreover, since $\left(\sigma, \sigma^{\prime}\right)$ belongs to $\operatorname{Inv}_{A} \operatorname{Polp}_{A}^{(<s)} Q$, we have $f \triangleright\left(\sigma, \sigma^{\prime}\right)$ for all $f \in \operatorname{Polp}_{A}^{(<s)} Q$. So, as $|B|<s$ and $B \subseteq \sigma$, we get $\mu_{B}^{\prime} \subseteq \sigma^{\prime}$. This proves $\left(\sigma, \sigma^{\prime}\right) \in s-\mathrm{LOC}_{A}[Q]_{\mathrm{Rp}_{A}}$.

Lemma 5.10. Any semiclone $F \subseteq \mathrm{O}_{A}$ satisfies $F^{(n)} \subseteq\left[F^{(s)}\right]_{\mathrm{O}_{A}}$ for all arities $n, s \in \mathbb{N}$ where $0<n \leq s$.

For $n=0<s$, the previous lemma (and its proof) fail. This is why in the following corollary to Theorem 5.9 , the arity 0 cannot be omitted in general.

Corollary 5.11. For $s \in \mathbb{N}$ and any set $Q \subseteq \mathrm{R}_{A}$ of relation pairs, we have the equality $\operatorname{Invp}_{A} \operatorname{Polp}_{A}^{(0, s)} Q=(s+1)-\mathrm{LOC}_{A}[Q]_{\mathrm{Rp}_{A}}$.

Corollary 5.12. For $s, m \in \mathbb{N}$ and any set $Q \subseteq \operatorname{Rp}_{A}$ such that $(\emptyset, \emptyset) \in Q^{(m)}$, we have $\operatorname{Invp}_{A} \operatorname{Polp}_{A}^{(s)} Q=(s+1)-\mathrm{LOC}_{A}[Q]_{\mathrm{Rp}_{A}}$.

Moreover, we can infer that a set $Q \subseteq \mathrm{Rp}_{A}$ is closed with respect to [ ] $\mathrm{Rp}_{A}$ and $s-\mathrm{LOC}_{A}$ if (and clearly only if) it is closed with respect to the operator $s-\mathrm{LOC}_{A}[]_{\mathrm{Rp}_{A}}$. This fact can be seen to generalise Proposition 3.8(ii),(iii) in $[30$, p. 30], where similar statements have been proven for relational clones. 
Corollary 5.13. For $0<s \leq \aleph_{0}$, a set $Q \subseteq \mathrm{Rp}_{A}$ of relation pairs is an $s$-locally closed relation pair clone if and only if $s-\mathrm{LOC}_{A}[Q]_{\mathrm{Rp}_{A}}=Q$.

Corollary 5.14 (cf. Proposition 3.9 in [30, p. 30]). For any $Q \subseteq \mathrm{Rp}_{A}$, we have $\operatorname{Polp}_{A}^{(<n)} Q=\operatorname{Polp}_{A}^{(<n)}[Q]_{\mathrm{Rp}_{A}}=\operatorname{Polp}_{A}^{(<n)} s-\mathrm{LOC}_{A}[Q]_{\mathrm{Rp}_{A}}$ for $0<n \leq s \leq \aleph_{0}$.

5.3. Characterisation of local closures for relation pairs. Finally, we shall consider another characterisation of the $s$-local closure operators, involving $s$-directed unions. The statement can be improved for sets of relation pairs fulfilling an additional closure property, which is in particular satisfied by relation pair clones. Hence, our characterisation is especially useful in connection with the operator $\aleph_{0}-\mathrm{LOC}_{A}[]_{\mathrm{Rp}_{A}}$.

Our result is a generalisation of Proposition 1.13 in [30, p. 18] to relation pairs (see also [29, Proposition 1.6, p. 256]). Without further assumptions on the set $Q$ of relation pairs, it works provided one accepts the axiom of choice.

Proposition 5.15. For any set $Q \subseteq \mathrm{Rp}_{A}$ of relation pairs and all cardinals $1<s \leq \aleph_{0}$, the following holds:

$$
s-\mathrm{LOC}_{A} Q=\biguplus_{m \in \mathbb{N}}\left\{\begin{array}{r|r}
\exists \sigma^{\prime \prime} \in \mathrm{R}_{A}^{(m)}: \sigma^{\prime \prime} \subseteq \sigma^{\prime} \wedge \\
\exists \mathcal{T} \subseteq \mathrm{Rp}_{A}^{(m)} \text { s-directed }: \\
\left(\sigma, \sigma^{\prime \prime}\right) \in \mathrm{Rp}_{A}^{(m)} & (\sigma \mathcal{T} \wedge \\
& \forall\left(\varrho, \varrho^{\prime}\right) \in \mathcal{T} \exists \tilde{\varrho} \in \mathrm{R}_{A}^{(m)}: \\
\varrho \subseteq \tilde{\varrho} \wedge\left(\tilde{\varrho}, \varrho^{\prime}\right) \in Q^{(m)}
\end{array}\right\} .
$$

If $Q \subseteq \mathrm{Rp}_{A}$ is closed under arbitrary intersections of pairs of identical arity and $s=\aleph_{0}$, the $\aleph_{0}$-directed collection $\mathcal{T}$ can be replaced by just a directed one.

Proof. To prove the inclusion " $\supseteq$ ", let us consider any $m \in \mathbb{N}$ and a pair $\left(\sigma, \sigma^{\prime}\right) \in \mathrm{Rp}_{A}^{(m)}$ satisfying the lengthy condition in the proposition. Its first part says that there is an $s$-directed system $\mathcal{T} \subseteq \mathrm{Rp}_{A}^{(m)}$ whose union equals $\left(\sigma, \sigma^{\prime \prime}\right)$ for some $m$-ary relation $\sigma^{\prime \prime} \subseteq \sigma^{\prime}$. The remaining part states that for every pair $\left(\varrho, \varrho^{\prime}\right) \in \mathcal{T}$, there is an $m$-ary relation $\varrho \supseteq \varrho$ such that $\left(\tilde{\varrho}, \varrho^{\prime}\right) \in Q^{(m)}$. This implies that $\left(\varrho, \varrho^{\prime}\right) \in \rightarrow\left\{\left(\tilde{\varrho}, \varrho^{\prime}\right)\right\} \leftarrow \subseteq \rightarrow Q^{\leftarrow} \subseteq \rightarrow s$-LOC ${ }_{A} Q^{\leftarrow}$. Since the set $s$-LOC $A$ is $s$-locally closed by Corollary 2.10 , it is also closed under relaxation. Hence, we have $\mathcal{T} \subseteq \rightarrow s$-LOC ${ }_{A} Q^{\leftarrow}=s$-LOC $A$. Now as $\mathcal{T}$ is an $s$-directed system, Lemma 2.13 yields that $\left(\sigma, \sigma^{\prime \prime}\right) \in s-\mathrm{LOC}_{A} Q$. Thus, from $\sigma^{\prime \prime} \subseteq \sigma^{\prime} \subseteq \sigma=\sigma$, we get

$$
\left(\sigma, \sigma^{\prime}\right) \in \rightarrow\left\{\left(\sigma, \sigma^{\prime \prime}\right)\right\}^{\leftarrow} \subseteq \rightarrow s-\mathrm{LOC}_{A} Q^{\leftarrow}=s-\mathrm{LOC}_{A} Q
$$

For the converse inclusion, take any $\left(\sigma, \sigma^{\prime}\right) \in s-\mathrm{LOC}_{A}^{(m)} Q, m \in \mathbb{N}$. Then for any $B \subseteq \sigma$ such that $|B|<s$, the set $\Sigma_{B}:=\left\{\left(\varrho, \varrho^{\prime}\right) \in Q^{(m)} \mid B \subseteq \varrho \wedge \varrho^{\prime} \subseteq \sigma^{\prime}\right\}$ is non-empty; using the axiom of choice, one can fix some pair $\left(\tilde{\varrho}_{B}, \varrho_{B}^{\prime}\right) \in \Sigma_{B}$. It satisfies $\varrho_{B}^{\prime} \subseteq \sigma^{\prime} \subseteq \sigma$; thus, $\varrho_{B}^{\prime} \subseteq \varrho_{B} \cap \sigma=: \varrho_{B}$. By construction, we have $B \subseteq \varrho_{B} \subseteq \tilde{\varrho}_{B}$; thus, putting $\mathcal{T}:=\left\{\left(\varrho_{B}, \varrho_{B}^{\prime}\right)|B \subseteq \sigma \wedge| B \mid<s\right\}$, the 
collection $\mathcal{T}$ satisfies the second part of the condition we need to verify. We shall check that $\mathcal{T}$ is $s$-directed farther below; first we deal with the union $\left(\mu, \mu^{\prime}\right):=\bigcup \mathcal{T}$ (meaning union in both components). Since for every subset $B \subseteq \sigma,|B|<s$, we have $\varrho_{B}^{\prime} \subseteq \sigma^{\prime}$ and $\varrho_{B} \subseteq \sigma$, it follows that also $\mu^{\prime} \subseteq \sigma^{\prime}$ and $\mu \subseteq \sigma$. Due to $s>1$, we have that $\sigma=\bigcup_{B \subseteq \sigma,|B|<s} B \subseteq \bigcup_{B \subseteq \sigma,|B|<s} \varrho_{B}=\mu$, wherefore $\mu=\sigma$. This shows that $\left(\sigma, \sigma^{\prime}\right)$ has the right form to fit into the set on the right-hand side, provided we establish that the non-empty set $\mathcal{T}$ is $s$-directed.

For this goal, we consider $t<s$ subsets $B_{0}, \ldots, B_{t-1} \subseteq \sigma$ subject to the condition $\left|B_{i}\right|<s$ for each $0 \leq i<t$ and tuples $r_{i} \in \varrho_{B_{i}} \subseteq \sigma$. Let us define $C:=\left\{r_{i} \mid 0 \leq i<t\right\} \subseteq \sigma$. As $|C| \leq t<s$, the pair $\left(\varrho_{C}, \varrho_{C}^{\prime}\right)$ belongs to $\mathcal{T}$ by definition. Thus, $C \subseteq \varrho_{C}$ demonstrates $s$-directedness, concluding the proof.

If $Q$ is closed with respect to intersections and $s=\aleph_{0}$, to prove the inclusion " $\subseteq$ ", one constructs the pair $\left(\tilde{\varrho}_{B}, \varrho_{B}^{\prime}\right)$ in $\Sigma_{B}$ as $\bigcap \Sigma_{B}$. Then $\mathcal{T}$ becomes directed.

Remark 5.16. The inclusion " $\subseteq$ " in Proposition 5.15 fails to hold for $s=1$. Consider, for example, any pair of relations $\varrho^{\prime} \subseteq \varrho \subsetneq A^{m}$ for some fixed $m \in \mathbb{N}$. Define $Q:=\rightarrow\left\{\left(\varrho, \varrho^{\prime}\right)\right\}^{\leftarrow}=\left\{\left(\sigma, \sigma^{\prime}\right) \in \operatorname{Rp}_{A}^{(m)} \mid \varrho^{\prime} \subseteq \sigma^{\prime} \subseteq \sigma \subseteq \varrho\right\}$; then $Q$ is certainly closed with respect to relaxation, and, moreover, it is not hard to see that it is also closed under arbitrary non-empty unions, i.e., 1-directed unions. Therefore, the set $U$ appearing on the right-hand side in Proposition 5.15 is contained in $Q$. Now, the set

$$
1-\mathrm{LOC}_{A} Q=\left\{\left(\sigma, \sigma^{\prime}\right) \in \operatorname{Rp}_{A}^{(m)} \mid \exists\left(\mu, \mu^{\prime}\right) \in Q^{(m)}: \sigma^{\prime} \supseteq \mu^{\prime}\right\}
$$

clearly contains $\left(A^{m}, \varrho^{\prime}\right)$, but $\left(A^{m}, \varrho^{\prime}\right) \notin U$ due to $A^{m} \nsubseteq \varrho$, i.e., $\left(A^{m}, \varrho^{\prime}\right) \notin Q$.

\section{Special cases}

6.1. Proper semiclones. Based on the results of the previous section, we may also characterise all $s$-locally closed semiclones that fail to be clones.

Proposition 6.1. For any cardinal $0<s \leq \aleph_{0}$ and any carrier set $A$, the collection $\left\{F \in \mathcal{S}_{A} \backslash \mathcal{L}_{A} \mid F=s-\mathrm{Loc}_{A} F\right\}$ can be relationally described in the form $\left\{\operatorname{Polp}_{A} Q \mid Q \subseteq \operatorname{Rp}_{A}^{(<s)} \wedge \exists\left(\varrho, \varrho^{\prime}\right) \in Q: \varrho^{\prime} \subsetneq \varrho\right\}$.

Using the theory of the previous sections, we can also prove a decidability result regarding the question if a clone with projections removed yields a semiclone, or if the non-trivial functions generate the projections. For this we need a more detailed analysis of the process generating $\Gamma_{F}(B)$ (cf. Lemma 5.1).

Lemma 6.2. Let $K$ be any set, $B \subseteq A^{K}$, and $F \subseteq \mathrm{O}_{A}$. Define $R_{0}:=B$ and

$$
\begin{aligned}
S_{j} & :=\biguplus_{n \in \mathbb{N}}\left\{f \circ\left(g_{0}, \ldots, g_{n-1}\right) \mid f \in F^{(n)} \wedge\left(g_{0}, \ldots, g_{n-1}\right) \in R_{j}{ }^{n}\right\}, \\
R_{j+1} & :=R_{j} \cup S_{j},
\end{aligned}
$$


for $j \in \mathbb{N}$; set $R:=\bigcup_{j \in \mathbb{N}} R_{j}$ and $S:=\bigcup_{j \in \mathbb{N}} S_{j}$. Employing the straightforward generalisation of the preservation concept to infinite arities, the pair $(R, S)$ is the least (with respect to $\leq$ ) member of the set

$$
Q_{B}:=\left\{\left(\varrho, \varrho^{\prime}\right) \mid B \cup \varrho^{\prime} \subseteq \varrho \subseteq A^{K},\left(\varrho, \varrho^{\prime}\right) \text { is preserved by all } f \in F\right\} .
$$

If $R_{n}=R_{n+1}$, i.e., $S_{n} \subseteq R_{n}$, holds for some $n \in \mathbb{N}$, then the equalities $S_{m}=S_{n}$ and $R_{m}=R_{n}$ hold for all $m \geq n$. Therefore, for finite $A$ and finite $K$, the condition $R_{n}=R_{n+1}$ is satisfied for some $n \leq\left|A^{K}\right|$.

Proof. First, we note that, by definition, $R_{j} \subseteq R_{j+1}$, which implies $S_{j} \subseteq S_{j+1}$, holds for all $j \in \mathbb{N}$. Hence, the unions defining $R$ and $S$ are directed.

It is not difficult to see that $(R, S)$ belongs to $Q_{B}$. Namely, we have that $B=R_{0} \subseteq R$ and $S_{j} \subseteq R_{j+1} \subseteq R$ for every $j \in \mathbb{N}$, whence $S \subseteq R$. To prove that $(R, S)$ is preserved by every $n$-ary $f \in F$, one considers an $n$-tuple of tuples $\left(g_{0}, \ldots, g_{n-1}\right) \in R^{n}$. Due to directedness of the union producing $R$ and finiteness of $n$, there exists one $j \in \mathbb{N}$ such that $\left(g_{0}, \ldots, g_{n-1}\right) \in R_{j}{ }^{n}$, wherefore $f \circ\left(g_{0}, \ldots, g_{n-1}\right) \in S_{j} \subseteq S$. Consequently, $(R, S)$ is preserved by every member of $F$ and thus belongs to $Q_{B}$.

Second, take any pair $\left(\varrho, \varrho^{\prime}\right) \in Q_{B}$. By definition, we have $R_{0}=B \subseteq \varrho$. Moreover, supposing that $R_{j} \subseteq \varrho$, by the preservation condition, we get that $S_{j} \subseteq \varrho^{\prime} \subseteq \varrho$ and hence $R_{j+1}=R_{j} \cup S_{j} \subseteq \varrho$, as well as $S_{j} \subseteq \varrho^{\prime}$. Thus, by induction, we have shown $R=\bigcup_{j \in \mathbb{N}} R_{j} \subseteq \varrho$ and $S=\bigcup_{j \in \mathbb{N}} S_{j} \subseteq \varrho^{\prime}$. This proves that $(R, S) \leq\left(\varrho, \varrho^{\prime}\right)$, whence $(R, S)$ is the $\leq$-least member of $Q_{B}$.

By induction, it is easy to see that $R_{n}=R_{n+1}$ entails $S_{m}=S_{n}$ and $R_{m}=R_{n}$ for all $m \geq n$. Moreover, if $R_{0}, R_{1}, \ldots, R_{n}$ are pairwise distinct (i.e., form a strictly increasing chain $\left.R_{0} \subsetneq R_{1} \subsetneq \cdots \subsetneq R_{n}\right)$, then $n \leq\left|R_{n}\right| \leq\left|A^{K}\right|$. Thus, for finite $A$ and $K, R_{n}=R_{n+1}$ must hold for some $n \leq\left|A^{K}\right|$.

The following lemma goes back to an idea of Peter Mayr.

Lemma 6.3. For $F \subseteq \mathrm{O}_{A}$, we have $\left[F \backslash \mathrm{J}_{A}\right]_{\mathrm{O}_{A}}=\left[\langle F\rangle_{\mathrm{O}_{A}} \backslash \mathrm{J}_{A}\right]_{\mathrm{O}_{A}}$.

Proof. Using Lemma 3.5(b), we have

$$
\begin{aligned}
& F \backslash \mathrm{J}_{A} \subseteq\langle F\rangle_{\mathrm{O}_{A}} \backslash \mathrm{J}_{A}=\left\langle F \backslash \mathrm{J}_{A}\right\rangle_{\mathrm{O}_{A}} \backslash \mathrm{J}_{A}=\left(\left[F \backslash \mathrm{J}_{A}\right]_{\mathrm{O}_{A}} \cup \mathrm{J}_{A}\right) \backslash \mathrm{J}_{A} \\
& =\left[F \backslash \mathrm{J}_{A}\right]_{\mathrm{O}_{A}} \backslash \mathrm{J}_{A} \subseteq\left[F \backslash \mathrm{J}_{A}\right]_{\mathrm{O}_{A}},
\end{aligned}
$$

whence $\left[F \backslash \mathrm{J}_{A}\right]_{\mathrm{O}_{A}}=\left[\langle F\rangle_{\mathrm{O}_{A}} \backslash \mathrm{J}_{A}\right]_{\mathrm{O}_{A}}$ by another application of []$_{\mathrm{O}_{A}}$.

We can now prove the problem of whether a finitely generated clone on a finite set generates projections from its non-trivial members to be decidable.

Proposition 6.4. For both $A$ and $F \subseteq \mathrm{O}_{A}$ finite, it is decidable whether $\langle F\rangle_{\mathrm{O}_{A}} \backslash \mathrm{J}_{A} \in \mathcal{S}_{A}$.

Proof. Since $\langle F\rangle_{\mathrm{O}_{A}}$ is a clone, and thus, in particular, a semiclone, containing $\langle F\rangle_{\mathrm{O}_{A}} \backslash \mathrm{J}_{A}$, we have $\langle F\rangle_{\mathrm{O}_{A}} \backslash \mathrm{J}_{A} \subseteq\left[\langle F\rangle_{\mathrm{O}_{A}} \backslash \mathrm{J}_{A}\right]_{\mathrm{O}_{A}} \subseteq\langle F\rangle_{\mathrm{O}_{A}}$. Therefore, by Lemma 3.5(c), the conditions $\langle F\rangle_{\mathrm{O}_{A}} \backslash \mathrm{J}_{A} \notin \mathcal{S}_{A},\langle F\rangle_{\mathrm{O}_{A}} \backslash \mathrm{J}_{A} \subsetneq\left[\langle F\rangle_{\mathrm{O}_{A}} \backslash \mathrm{J}_{A}\right]_{\mathrm{O}_{A}}$, 
$\left[\langle F\rangle_{\mathrm{O}_{A}} \backslash \mathrm{J}_{A}\right]_{\mathrm{O}_{A}} \cap \mathrm{J}_{A} \neq \emptyset, \mathrm{J}_{A} \subseteq\left[\langle F\rangle_{\mathrm{O}_{A}} \backslash \mathrm{J}_{A}\right]_{\mathrm{O}_{A}}$, and id $\sin _{A} \in\left[\langle F\rangle_{\mathrm{O}_{A}} \backslash \mathrm{J}_{A}\right]_{\mathrm{O}_{A}}^{(1)}$ are all equivalent. By Lemma 6.3, $\left[\langle F\rangle_{\mathrm{O}_{A}} \backslash \mathrm{J}_{A}\right]_{\mathrm{O}_{A}}^{(1)}=\left[F \backslash \mathrm{J}_{A}\right]_{\mathrm{O}_{A}}^{(1)}$, and using Corollary 5.2 for $n=1, X=A$, and some bijection $\beta$ between $A$ and its cardinality, we have a description of the invariant pair $\left(\varrho_{A, 1}, \varrho_{A, 1}^{\prime}\right)=\Gamma_{F \backslash \mathrm{J}_{A}}\left(\left\{\operatorname{id}_{A} \circ \beta\right\}\right)$. Via Lemma 6.2 , this invariant relation pair generated by $\operatorname{id}_{A} \circ \beta$ can be expressed as $\left(\bigcup_{j \in \mathbb{N}} R_{j}, \bigcup_{j \in \mathbb{N}} S_{j}\right)$ and finiteness of $A$ guarantees that $R_{n}=R_{n+1}$ happens for some $n \leq\left|A^{A}\right|$. This implies $\left\{f \circ \beta \mid f \in\left[F \backslash \mathrm{J}_{A}\right]_{\mathrm{O}_{A}}^{(1)}\right\}=\varrho_{A, 1}^{\prime}$ can be written as the finite union $U:=\bigcup_{0 \leq j \leq\left|A^{A}\right|} S_{j}$, which due to finiteness of $F$, can be straightforwardly calculated using the definitions of Lemma 6.2. Hence, one may check if $\operatorname{id}_{A}$ is in $\left[F \backslash \mathrm{J}_{A}\right]_{\mathrm{O}_{A}}^{(1)}$ by checking if $\beta=\operatorname{id}_{A} \circ \beta \in U$.

6.2. Closed transformation semigroups. By considering just unary parts, we can characterise $s$-locally closed (proper) transformation semigroups.

Proposition 6.5. For $0<s \leq \aleph_{0}$ and a set $H \subseteq \mathrm{O}_{A}^{(1)}$ of transformations, the following facts are equivalent.

(a) $H$ is an s-locally closed transformation semigroup ( and $\operatorname{id}_{A} \notin H$ ).

(b) $H=\operatorname{Polp}_{A}^{(1)} \operatorname{Invp}_{A}^{(<s)} H$ (and there is $\left(\varrho, \varrho^{\prime}\right) \in \operatorname{Invp}_{A}^{(<s)} H$ with $\left.\varrho^{\prime} \neq \varrho\right)$.

(c) $H=\operatorname{Polp}_{A}^{(1)} Q$ for some $Q \subseteq \operatorname{Rp}_{A}^{(<s)}$ (where $\varrho \neq \varrho^{\prime}$ for some $\left(\varrho, \varrho^{\prime}\right) \in Q$ ). For $A \neq \emptyset$ and $s<\aleph_{0}$, the arity restrictions " $<s$ " can be replaced by " $s-1$ ".

By intersecting (in a similar way as outlined in this subsection) with other classes of functions, for example, the set of all permutations instead of all unary operations, one can obtain further characterisations of $s$-locally closed classes of functions in terms of relation pairs. Continuing the example of permutations, one may get a characterisation of all $s$-locally closed (proper) transformation semigroups that consist of permutations only. As on finite carrier sets, every permutation has a finite order; such a result is necessarily more appealing on infinite domains.

6.3. Classical Pol-Inv Galois correspondence. Here we demonstrate that it is not difficult to derive the characterisations of the closure operators of the Galois connection given by polymorphisms and invariant relations from our theorems above. In this respect, we first consider the framework including nullary operations and relations, as discussed in [3]; from there, it will be a small step to obtain the variants known from [30, 29].

First, we recollect information concerning the relationship of the operators $\mathrm{Pol}_{A}$ and $\operatorname{Inv}_{A}$ with respect to $\mathrm{Polp}_{A}$ and $\operatorname{Invp}_{A}$ (cf. before Lemma 2.3).

Lemma 6.6. For $Q \subseteq \mathrm{R}_{A}, F \subseteq \mathrm{O}_{A}$ and any $n \in \mathbb{N}$, we have

$$
\begin{array}{r}
\operatorname{Pol}_{A}^{(n)} Q=\operatorname{Polp}_{A}^{(n)} \biguplus_{m \in \mathbb{N}}\left\{(\varrho, \varrho) \mid \varrho \in Q^{(m)}\right\}, \\
\operatorname{Inv}_{A}^{(n)} F=\left\{\varrho \mid(\varrho, \varrho) \in \operatorname{Invp}_{A}^{(n)} F\right\} .
\end{array}
$$


Lemma 6.7. Suppose a set $F \subseteq \mathrm{O}_{A}$ of operations satisfies $F \cap \mathrm{J}_{A} \neq \emptyset$; then $\operatorname{Invp}_{A} F=\biguplus_{m \in \mathbb{N}}\left\{(\varrho, \varrho) \mid \varrho \in \operatorname{Inv}_{A}^{(m)} F\right\}$.

This enables us now to derive the characterisation of the closure operators $\mathrm{Pol}_{A} \operatorname{Inv}_{A}^{(<s)}$ and $\mathrm{Pol}_{A} \operatorname{Inv}_{A}^{(s)}$. The case $s=\aleph_{0}$ yields [3, Theorem 3.17, p. 29].

Theorem 6.8. We have $s-\operatorname{Loc}_{A}\langle F\rangle_{\mathrm{O}_{A}}=\operatorname{Pol}_{A} \operatorname{Inv}_{A}^{(<s)} F$ for any $F \subseteq \mathrm{O}_{A}$ and $0<s \leq \aleph_{0}$; if $s<\aleph_{0}$, then the equality $s-\operatorname{Loc}_{A}\langle F\rangle_{\mathrm{O}_{A}}=\operatorname{Pol}_{A} \operatorname{Inv}_{A}^{(s-1)} F$ holds.

Proof. Using Lemma 3.5(b), we can write $\langle F\rangle_{\mathrm{O}_{A}}=\left[F \cup\left\{\operatorname{id}_{A}\right\}\right]_{\mathrm{O}_{A}}$; hence, Theorem 5.3 entails that $s-\operatorname{Loc}_{A}\langle F\rangle_{\mathrm{O}_{A}}=s-\operatorname{Loc}_{A}\left[F \cup\left\{\operatorname{id}_{A}\right\}\right]_{\mathrm{O}_{A}}$ coincides with $\operatorname{Polp}_{A} \operatorname{Invp}_{A}^{(<s)}\left(F \cup\left\{\operatorname{id}_{A}\right\}\right)$, which by Lemma 6.7, equals

$$
\begin{aligned}
& \operatorname{Polp}_{A} \biguplus_{0 \leq m<s}\left\{(\varrho, \varrho) \mid \varrho \in \operatorname{Inv}_{A}^{(m)}\left(F \cup\left\{\operatorname{id}_{A}\right\}\right)\right\}
\end{aligned}
$$

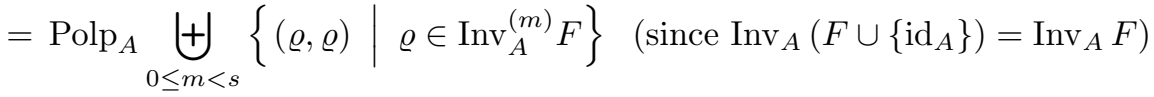

$$
\begin{aligned}
& =\bigcap_{0 \leq m<s} \operatorname{Polp}_{A}\left\{(\varrho, \varrho) \mid \varrho \in \operatorname{Inv}_{A}^{(m)} F\right\} \\
& =\bigcap_{0 \leq m<s} \operatorname{Pol}_{A} \operatorname{Inv}_{A}^{(m)} F=\operatorname{Pol}_{A} \operatorname{Inv}_{A}^{(<s)} F \text {. }
\end{aligned}
$$

For $A \neq \emptyset$, we may replace Theorem 5.3 by Corollary 5.5 and therefore the operator $\operatorname{Polp}_{A} \operatorname{Invp}_{A}^{(<s)}$ by $\operatorname{Polp}_{A} \operatorname{Invp}_{A}^{(s-1)}$. The rest of the argument is analogous to the above. For $A=\emptyset, \mathrm{Pol}_{A} \operatorname{Inv}_{A}^{(s-1)} F$ and $\operatorname{Pol}_{A} \operatorname{Inv}_{A}^{(<s)}=s-\operatorname{Loc}_{A}\langle F\rangle_{\mathrm{O}_{A}}$ are both clones on $\emptyset$, but there exists only one such structure, namely $\mathrm{O}_{\emptyset}$.

In contrast to semiclones, nullary relations are never needed to discern locally closed clones. In fact, invariants of small arity may always be neglected.

Corollary 6.9. For a set of operations $F \subseteq \mathrm{O}_{A}$ and any arity $m \in \mathbb{N}$, we have the equality $\mathrm{Pol}_{A} \operatorname{Inv}_{A}^{(\geq m)} F=\operatorname{Pol}_{A} \operatorname{Inv}_{A} F=\aleph_{0}-\operatorname{Loc}_{A}\langle F\rangle_{\mathrm{O}_{A}}$.

Lemma 6.10. For operations $F \subseteq \mathrm{O}_{A} \backslash \mathrm{O}_{A}^{(0)}$ of positive arity and every cardinal $0<s \leq \aleph_{0}$, the equality $s-\operatorname{Loc}_{A}^{(0)}\langle F\rangle_{\mathrm{O}_{A}}=\langle F\rangle_{\mathrm{O}_{A}}^{(0)}=\emptyset$ holds.

Corollary 6.11. Let $F \subseteq \mathrm{O}_{A} \backslash \mathrm{O}_{A}^{(0)}$ be without nullary operations and $s \in \mathbb{N}_{+}$; then the equalities $\mathrm{Pol}_{A}^{(>0)} \operatorname{Inv}_{A} F=\operatorname{Pol}_{A}^{(>0)} \operatorname{Inv}_{A}^{(>0)} F=\aleph_{0}-\operatorname{Loc}_{A}\langle F\rangle_{\mathrm{O}_{A}}$ and $\mathrm{Pol}_{A}^{(>0)} \operatorname{Inv}_{A}^{(s-1)} F=s-\operatorname{Loc}_{A}\langle F\rangle_{\mathrm{O}_{A}}$ are fulfilled.

For $F \subseteq \mathrm{O}_{A} \backslash \mathrm{O}_{A}^{(0)}$, the equalities $\aleph_{0}-\operatorname{Loc}_{A}\langle F\rangle_{\mathrm{O}_{A}}=\operatorname{Pol}_{A}^{(>0)} \operatorname{Inv}_{A}^{(>0)} F$ as well as $s-\operatorname{Loc}_{A}\langle F\rangle_{\mathrm{O}_{A}}=\mathrm{Pol}_{A}^{(>0)} \operatorname{Inv}_{A}^{(s-1)} F$ for $s>1$ express two of the main results regarding Pol - Inv that one finds in [29, Theorem 3.2, p. 260], [30, Theorem 4.1, p. 31], where neither nullary operations nor nullary relations were considered.

In order to attack the relational side of the Pol - Inv Galois correspondence, we need to express generated relational clones, i.e., the closure []$_{\mathrm{R}_{A}}$ of a set 
of relations under general superpositions, in terms of generated relation pair clones. This is prepared in the following lemma.

Lemma 6.12. For any set $Q \subseteq \mathrm{R}_{A}$, we have

$$
\biguplus_{m \in \mathbb{N}}\left\{(\varrho, \varrho) \mid \varrho \in[Q]_{\mathrm{R}_{A}}^{(m)}\right\}=\left[\biguplus_{m \in \mathbb{N}}\left\{(\varrho, \varrho) \mid \varrho \in Q^{(m)}\right\}\right]_{\mathrm{Rp}_{A}} .
$$

Lemma 6.13. For any set $Q \subseteq \mathrm{R}_{A}$ and $1<s \leq \aleph_{0}$, we have

$$
s-\mathrm{LOC}_{A} \biguplus_{m \in \mathbb{N}}\left\{(\varrho, \varrho) \mid \varrho \in Q^{(m)}\right\}=\biguplus_{m \in \mathbb{N}}\left\{(\sigma, \sigma) \mid \sigma \in s-\mathrm{L} \varnothing \mathrm{C}_{A}^{(m)} Q\right\} .
$$

Theorem 6.14 (see [3, Theorem 3.20, p. 31] for $s=\aleph_{0}$ ). For any cardinal $0<s \leq \aleph_{0}$ and any set $Q \subseteq \mathrm{R}_{A}$, we have $s-\mathrm{L}_{A}[Q]_{\mathrm{R}_{A}}=\operatorname{Inv}_{A} \operatorname{Pol}_{A}^{(<s)} Q$; if $s<\aleph_{0}$, then $\operatorname{Inv}_{A} \mathrm{Pol}_{A}^{(<s)} Q$ can be replaced by $\operatorname{Inv}_{A} \operatorname{Pol}_{A}^{(0, s-1)} Q$.

Proof. Using the previous results, we calculate for $Q \subseteq \mathrm{R}_{A}$ and $1<s \leq \aleph_{0}$ :

$$
\begin{aligned}
\operatorname{Inv}_{A} \operatorname{Pol}_{A}^{(<s)} Q & \stackrel{6.6}{=} \operatorname{Inv}_{A} \operatorname{Polp}_{A}^{(<s)} \biguplus_{m \in \mathbb{N}}\left\{(\varrho, \varrho) \mid \varrho \in Q^{(m)}\right\} \\
& \stackrel{6.6}{=}\left\{\sigma \mid(\sigma, \sigma) \in \operatorname{Invp}_{A} \operatorname{Polp}_{A}^{(<s)} \biguplus_{m \in \mathbb{N}}\left\{(\varrho, \varrho) \mid \varrho \in Q^{(m)}\right\}\right\} \\
& \stackrel{5.9}{=}\left\{\sigma \mid(\sigma, \sigma) \in s-\operatorname{LOC}_{A}\left[\biguplus_{m \in \mathbb{N}}\left\{(\varrho, \varrho) \mid \varrho \in Q^{(m)}\right\}\right]_{\mathrm{Rp}_{A}}\right\} \\
& \stackrel{6.12}{=}\left\{\sigma \mid(\sigma, \sigma) \in s-\mathrm{LOC}_{A} \biguplus_{m \in \mathbb{N}}\left\{(\varrho, \varrho) \mid \varrho \in[Q]_{\mathrm{R}_{A}}^{(m)}\right\}\right\} \\
& \stackrel{6.13}{=}\left\{\sigma \mid(\sigma, \sigma) \in \biguplus_{m \in \mathbb{N}}\left\{(\varrho, \varrho) \mid \varrho \in s-\mathrm{L} \varnothing \mathrm{C}_{A}^{(m)}[Q]_{\mathrm{R}_{A}}\right\}\right\} \\
& =s-\mathrm{L} \varnothing \mathrm{C}_{A}[Q]_{\mathrm{R}_{A}} .
\end{aligned}
$$

Invoking Corollary 5.11 instead of Theorem 5.9 in the previous calculation, we may replace the operator $\operatorname{Inv}_{A} \mathrm{Pol}_{A}^{(<s)}$ by $\operatorname{Inv}_{A} \mathrm{Pol}_{A}^{(0, s-1)}$, and $\operatorname{Invp}_{A} \operatorname{Polp}_{A}^{(<s)}$ by $\operatorname{Invp}_{A} \operatorname{Polp}_{A}^{(0, s-1)}$, respectively, in the manipulations above.

Due to inapplicability of Lemma 6.13 for $s=1$, this case needs a manual proof. Clearly, we have 1-L $\varnothing \mathrm{C}_{A}[Q]_{\mathrm{R}_{A}}=\left\{\sigma \in \mathrm{R}_{A} \mid \exists \varrho \in[Q]_{\mathrm{R}_{A}}: \sigma \supseteq \varrho\right\}$ and $\operatorname{Inv}_{A} \mathrm{Pol}_{A}^{(0)} Q=\operatorname{Inv}_{A}\left\{c_{a}^{(0)} \mid \forall \varrho \in Q:(a, \ldots, a) \in \varrho\right\}=\left\{\sigma \in \mathrm{R}_{A} \mid \sigma \supseteq \mu\right\}$, in which $\mu:=\{(a, \ldots, a) \mid a \in A \wedge \forall \varrho \in Q:(a, \ldots, a) \in \varrho\}$ and $c_{a}^{(0)}$ denotes the nullary operation with image $\{a\}$. It is easy to see that $\mu \in[Q]_{\mathrm{R}_{A}}$, namely, for $\sigma \in \mathrm{R}_{A}$, let $\beta$ : $\operatorname{ar}(\sigma) \rightarrow 1$ and $\alpha_{\varrho}:$ ar $(\varrho) \rightarrow 1$ for $\varrho \in Q$ be the unique constant mappings, then $\mu=\bigwedge_{\left(\alpha_{\varrho}\right)_{\varrho \in Q}}^{\beta}(\varrho)_{\varrho \in Q} \in[Q]_{\mathrm{R}_{A}}$. This proves the inclusion $\operatorname{Inv}_{A} \mathrm{Pol}_{A}^{(0)} Q \subseteq 1-\mathrm{L} \varnothing \mathrm{C}_{A}[Q]_{\mathrm{R}_{A}}$. The converse is simple: if $\sigma \in \mathrm{R}_{A}$ includes 
some $\varrho \in[Q]_{\mathrm{R}_{A}}$, and $c_{a}^{(0)} \in \operatorname{Pol}_{A}^{(0)} Q=\operatorname{Pol}_{A}^{(0)}[Q]_{\mathrm{R}_{A}}$, then $(a, \ldots, a) \in \varrho \subseteq \sigma$. As this holds for all constants in $\operatorname{Pol}_{A}^{(0)} Q$, we obtain $\sigma \in \operatorname{Inv}_{A} \operatorname{Pol}_{A}^{(0)} Q$.

Lemma 6.15. We have $\mathrm{Pol}_{A}\{\emptyset\}=\mathrm{O}_{A} \backslash \mathrm{O}_{A}^{(0)}$ and thereby $\mathrm{Pol}_{A}^{(s)}\{\emptyset\}=\mathrm{O}_{A}^{(s)}$ whenever $s \in \mathbb{N}_{+}$; therefore, $\operatorname{Pol}_{A}^{(s)}(Q \cup\{\emptyset\})=\operatorname{Pol}_{A}^{(s)} Q$ for any $Q \subseteq \mathrm{R}_{A}$.

Corollary 6.16. Let $Q \subseteq \mathrm{R}_{A}$ and $s \in \mathbb{N}$. Then $s-\mathrm{LOC}_{A}[Q]_{\mathrm{R}_{A}}=\operatorname{Inv}_{A} \operatorname{Pol}_{A}^{(s)} Q$ if (and, provided that $s>0$, also only if) $\emptyset \in \operatorname{Inv}_{A} \mathrm{Pol}_{A} Q$ (which is true in particular if $\emptyset \in Q)$.

Corollary 6.17. We have $\mathrm{LOC}_{A}[Q \cup\{\emptyset\}]_{\mathrm{R}_{A}}=\operatorname{Inv}_{A} \operatorname{Pol}_{A}^{(>0)} Q$ for $Q \subseteq \mathrm{R}_{A}$. Moreover, for $s \in \mathbb{N}_{+}$, the equality $s-\mathrm{LOC}_{A}[Q \cup\{\emptyset\}]_{\mathrm{R}_{A}}=\operatorname{Inv}_{A} \operatorname{Pol}_{A}^{(s)} Q$ holds.

Restricting the statement of Corollary 6.17 to sets $Q \subseteq \mathrm{R}_{A} \backslash \mathrm{R}_{A}^{(0)}$ and intersecting the equalities on both sides with $\mathrm{R}_{A} \backslash \mathrm{R}_{A}^{(0)}$ yields the characterisations $\operatorname{LOC}_{A}\left([Q \cup\{\emptyset\}]_{\mathrm{R}_{A}}^{(>0)}\right)=\operatorname{Inv}_{A}^{(>0)} \operatorname{Pol}_{A}^{(>0)} Q$ and, for positive parameters $s, s-\mathrm{LOC}_{A}\left([Q \cup\{\emptyset\}]_{\mathrm{R}_{A}}^{(>0)}\right)=\operatorname{Inv}_{A}^{(>0)} \operatorname{Pol}_{A}^{(s)} Q$. The closure $[Q \cup\{\emptyset\}]_{\mathrm{R}_{A}}^{(>0)}$ describes the appropriate notion of generated relational clone (as employed e.g., in [30]) if one does neither consider nullary operations nor relations in connection with Pol-Inv. With the two stated equalities, we have therefore established the two main results (see Theorem 4.2, p. 32, and Theorem 3.3, p. 260, respectively) of $[30,29]$ regarding the relational side of Pol - Inv.

\section{Possible applications}

In the literature, the Pol - Inv Galois connection has been very successfully employed to discover the structure of the lattice of all clones (e.g., [32, 23, 38, $37]$ ), but it is also fundamentally involved in investigating other problems in algebra and theoretical computer science $([11,2,1,7,36])$. It is to be expected that the theory developed within this article will find similar applications with respect to semiclones in the future, especially regarding infinite carrier sets.

In this connection, we briefly outline one possible idea, picking up again the topic of topologically closed (proper) transformation semigroups from the previous section. According to Proposition 6.5, for any set $Q \subseteq \operatorname{Rp}_{A}$ of relation pairs, the set of all locally closed transformation semigroups $S \subseteq \mathrm{O}_{A}^{(1)}$ lying properly below $\operatorname{Polp}_{A}^{(1)} Q$ can be described as all those sets $S=\operatorname{Polp}_{A}^{(1)} \Sigma$ (for $\left.\Sigma \subseteq \mathrm{Rp}_{A}\right)$ satisfying $\operatorname{Polp}_{A}^{(1)} \Sigma \subsetneq \operatorname{Polp}_{A}^{(1)} Q$. If $S$ is a maximal member of this collection with respect to inclusion, then $Q \subseteq \operatorname{Invp}_{A} \operatorname{Polp}_{A}^{(1)} Q \subsetneq \operatorname{Invp}_{A} S$. One may take any pair $\left(\varrho, \varrho^{\prime}\right) \in \operatorname{Invp}_{A} S \backslash \operatorname{Invp}_{A} \operatorname{Polp}_{A}^{(1)} Q$ and obtain that

$$
S=\operatorname{Polp}_{A}^{(1)} \operatorname{Invp}_{A} S \subseteq \operatorname{Polp}_{A}^{(1)}\left(Q \cup\left\{\left(\varrho, \varrho^{\prime}\right)\right\}\right) \subsetneq \operatorname{Polp}_{A}^{(1)} Q,
$$

which by maximality of $S$ entails that $S=\operatorname{Polp}_{A}^{(1)}\left(Q \cup\left\{\left(\varrho, \varrho^{\prime}\right)\right\}\right)$. In case that $\operatorname{Polp}_{A}^{(1)} Q$ is a monoid, i.e., $Q$ contains only relation pairs with identical 
components and $\operatorname{Polp}_{A} Q$ is a real clone, then one may also be interested in the maximal locally closed proper transformation semigroups below it. This additional requirement enforces that the pair $\left(\varrho, \varrho^{\prime}\right)$ one had to add above even has to be a proper relation pair, i.e., $\varrho^{\prime} \subsetneq \varrho$.

In a similar way, all maximal $s$-locally closed (possibly proper) semiclones (or $s$-locally closed transformation semigroups) below one specific structure of the respective sort can be described by preserving one additional relation pair. It is plausible that for certain sets $Q$, a complete characterisation in analogy to [32] can be attempted. Furthermore, on infinite carrier sets, the machinery developed in this paper can also be useful to reveal counterexamples, e.g., structures having no maximal proper ( $s$-locally closed) substructures below them. It is, for example, not hard to prove for $Q=\emptyset$, that proper semigroups of the form $\operatorname{Polp}_{A}^{(1)}\left\{\left(\varrho, \varrho^{\prime}\right)\right\}$ with $\varrho^{\prime} \subsetneq \varrho \subseteq A$ can never be maximal among all locally closed proper transformation semigroups on $A$ whenever $|A| \geq 2$.

The author is, moreover, confident that a generalisation of the presented theory to categories with finite powers is possible along the lines of [24], where a similar project has been realised for clones and the Pol-Inv Galois connection (at the same time, dualising the involved notions, which is not in our focus). Most of our results do not impose any restrictions on the carrier set, i.e., the particular object of the category of sets the Galois theory is based on. Therefore, the main theorems of this article could be a guideline and used to hint at what form of results to expect in the general setting. Once such a generalisation has been established, the corresponding results can be instantiated in any category of interest, as long as it has finite powers, for instance, in that of topological spaces. In this way, it may be possible to perform similar investigations as sketched above also for transformation semigroups consisting of continuous functions.

Acknowledgements. Open access funding provided by Austrian Science Fund (FWF). The author expresses his gratitude to Erhard Aichinger for an invitation to the Institute for Algebra at Johannes Kepler University Linz, which enabled fruitful discussions on some aspects of the topic with members of the institute including Erhard Aichinger, Peter Mayr, Keith Kearnes and Ágnes Szendrei. The author wishes to thank them, too, for their valuable comments and contributions. Moreover, he is grateful to both anonymous reviewers for careful proofreading, pointing out typos, suggesting an additional reference, and urging the author to develop a common framework for the local and $s$-local closure operators.

\section{REFERENCES}

[1] Barto, L.: The dichotomy for conservative constraint satisfaction problems revisited. In: 26th Annual IEEE Symposium on Logic in Computer Science-LICS 2011, pp. 301-310. IEEE Computer Soc., Los Alamitos, CA (2011)

[2] Barto, L., Kozik, M.: Absorbing subalgebras, cyclic terms, and the constraint satisfaction problem. Log. Methods Comput. Sci. 8 1:07, 27 pp. (2012) 
[3] Behrisch, M.: Clones with nullary operations. In: J. Power, C. Wingfield (eds.) Proceedings of the Workshop on Algebra, Coalgebra and Topology (WACT 2013), Electron. Notes Theor. Comput. Sci., vol. 303, pp. 3-35. Elsevier Sci. B. V., Amsterdam (2014)

[4] Behrisch, M.: Galois theory for semiclones. CoRR abs/1509.06355, 1-38 (2015). URL http://arxiv.org/abs/1509.06355v1

[5] Bodirsky, M., Pinsker, M.: Minimal functions on the random graph. Israel J. Math. 200, 251-296 (2014)

[6] Bodirsky, M., Pinsker, M.: Topological Birkhoff. Trans. Amer. Math. Soc. 367, 2527-2549 (2015)

[7] Bodirsky, M., Pinsker, M., Pongrácz, A.: Reconstructing the topology of clones. CoRR abs/1312.7699, 1-30 (2014)

[8] Bodnarčuk, V.G., Kalužnin, L.A., Kotov, V.N., Romov, B.A.: Galois theory for Post algebras. I. Cybernetics 5, 243-252 (1969)

[9] Bodnarčuk, V.G., Kalužnin, L.A., Kotov, V.N., Romov, B.A.: Galois theory for Post algebras. II. Cybernetics 5, 531-539 (1969)

[10] Bodnarčuk, V.G., Kalužnin, L.A., Kotov, V.N., Romov, В.А.: Теория Галуа для алгебр Поста. I, II [Galois theory for Post algebras. I, II]. Kibernetika (Kiev) 5, 1-10; ibid. 1-9 (1969)

[11] Bulatov, A.A.: A dichotomy theorem for constraint satisfaction problems on a 3-element set. J. ACM 53, 66-120 (2006)

[12] Couceiro, M.: On Galois connections between external operations and relational constraints: arity restrictions and operator decompositions. Acta Sci. Math. (Szeged) 7215-35 (2006)

[13] Couceiro, M., Foldes, S.: On closed sets of relational constraints and classes of functions closed under variable substitutions. Algebra Universalis 54, 149-165 (2005)

[14] Couceiro, M., Lehtonen, E.: Galois theory for sets of operations closed under permutation, cylindrification, and composition. Algebra Universalis 67, 273-297 (2012)

[15] Geiger, D.: Closed systems of functions and predicates. Pacific J. Math. 27, 95-100 (1968)

[16] Goldstern, M., Pinsker, M.: A survey of clones on infinite sets. Algebra Universalis 59, 365-403 (2008)

[17] Harnau, W.: Ein verallgemeinerter Relationen- und ein modifizierter Superpositionsbegriff für die Algebra der mehrwertigen Logik. [A generalised notion of relation and a modified notion of superposition for the algebra of multiple-valued logic.]. Habilitation thesis, Universität Rostock (1983)

[18] Harnau, W.: Ein verallgemeinerter Relationenbegriff für die Algebra der mehrwertigen Logik. I. Grundlagen [A generalised notion of relation for the algebra of multiple-valued logic. I. Foundations.]. Rostock. Math. Kolloq. 28, 5-17 (1985)

[19] Harnau, W.: Ein verallgemeinerter Relationenbegriff für die Algebra der mehrwertigen Logik. II. Relationenpaare [A generalised notion of relation for the algebra of multiple-valued logic. II. Pairs of relations.]. Rostock. Math. Kolloq. 31, 11-20 (1987)

[20] Harnau, W.: Ein verallgemeinerter Relationenbegriff für die Algebra der mehrwertigen Logik. III. Beweis [A generalised notion of relation for the algebra of multiple-valued logic. III. Proof.]. Rostock. Math. Kolloq. 32, 15-24 (1987)

[21] Jablonskij, S.V.: Функциональные построения в $k$-значной логике [Functional constructions in $k$-valued logic]. Trudy Mat. Inst. Steklov. 51, 5-142 (1958)

[22] Jablonskij, S.V., Gavrilov, G.P., Kudrjavcev, V.В.: Функции алгебры логики и классы Поста [Functions of algebraic logic and Post classes]. Izdat. "Nauka", Moscow (1966)

[23] Janov, Ju.I., Mučnik, A.А.: О существовании $k$-значных замкнутых классов, не имеющих конечного базиса [Existence of $k$-valued closed classes without a finite basis]. Dokl. Akad. Nauk SSSR 127, 44-46 (1959)

[24] Kerkhoff, S.: A general Galois theory for operations and relations in arbitrary categories. Algebra Universalis 68, 325-352 (2012) 
[25] Lau, D.: Function algebras on finite sets. A basic course on many-valued logic and clone theory. Springer Monographs in Mathematics. Springer, Berlin (2006)

[26] Lehtonen, E.: Closed classes of functions, generalized constraints, and clusters. Algebra Universalis 63, 203-234 (2010)

[27] Mal'cev, A.I.: Итеративные алгебры и многообразия Поста [Iterative algebras and Post varieties]. Algebra i Logika Sem. 5, 5-24 (1966)

[28] Pippenger, N.: Galois theory for minors of finite functions. Discrete Math. 254, 405-419 (2002)

[29] Pöschel, R.: Concrete representation of algebraic structures and a general Galois theory. In: Contributions to general algebra (Proc. Klagenfurt Conf., Klagenfurt, 1978), pp. 249-272. Heyn, Klagenfurt (1979)

[30] Pöschel, R.: A general Galois theory for operations and relations and concrete characterization of related algebraic structures, Report 1980, vol. 1. Akademie der Wissenschaften der DDR Institut für Mathematik, Berlin (1980). With German and Russian summaries

[31] Pöschel, R., Kalužnin, L.A.: Funktionen- und Relationenalgebren. Ein Kapitel der diskreten Mathematik, Mathematische Monographien, vol. 15. VEB Deutscher Verlag der Wissenschaften, Berlin (1979)

[32] Rosenberg, I.G.: Über die funktionale Vollständigkeit in den mehrwertigen Logiken. Struktur der Funktionen von mehreren Veränderlichen auf endlichen Mengen [On functional completeness in multiple-valued logics. Structure of functions of several variables on finite sets], Rozpravy Československé Akademie Věd: Řada matematických a přírodních věd, vol. 80. Academia (1970)

[33] Schmidt, J.: Clones and semiclones of operations. In: Universal algebra (Esztergom, 1977), Colloq. Math. Soc. János Bolyai, vol. 29, pp. 705-723. North-Holland, Amsterdam (1982)

[34] Szabó, L.: Concrete representation of related structures of universal algebras. I. Acta Sci. Math. (Szeged) 40, 175-184 (1978)

[35] Szendrei, Á.: Clones in universal algebra, Séminaire de Mathématiques Supérieures, vol. 99. Presses de l'Université de Montréal, Montreal, QC (1986)

[36] Vargas, E.: Clausal relations and $C$-clones. Discuss. Math. Gen. Algebra Appl. 30, $147-171$ (2010)

[37] Zhuk, D.N.: The lattice of the clones of self-dual functions in three-valued logic. In: Proceedings of the symposium held in Tuusula, May 23-25, 2011, 41st IEEE International Symposium on Multiple-Valued Logic ISMVL 2011, pp. 193-197. IEEE Computer Society, Los Alamitos, CA (2011)

[38] Zhuk, D.N.: The cardinality of the set of all clones containing a given minimal clone on three elements. Algebra Universalis 68, 295-320 (2012)

\section{Mike Behrisch}

Institut für Computersprachen, (currently at Institut für Diskrete Mathematik und Geometrie), Technische Universität Wien, A-1040 Vienna, Austria

e-mail: behrisch@logic.at

Open Access This article is distributed under the terms of the Creative Commons Attribution 4.0 International License (http://creativecommons.org/licenses/by/4.0/), which permits unrestricted use, distribution, and reproduction in any medium, provided you give appropriate credit to the original author(s) and the source, provide a link to the Creative Commons license, and indicate if changes were made. 